\title{
A Low Complexity and Effective Rapid Channel Tracking Scheme for UWB Multiple-Access Systems
}

\author{
Ho-Lung Hung • Chia-Hsin Cheng
}

Published online: 11 December 2013

(C) The Author(s) 2013. This article is published with open access at Springerlink.com

\begin{abstract}
In this paper, the applicability of the subspace-based blind adaptive algorithm in multiple access ultra-wideband systems is investigated. However, in the multiuser transmission environment, multiple access interference becomes a serious issue and results in the degradation of system performance. In order to overcome this shortcoming, we propose a novel and low complexity decision mechanism, termed the decision timing instant (DTI). A major advantage of the DTI algorithm is that it admits an adaptive implementation with low computational complexity instead of singular value decomposition. In the present paper, we exploit the joint blind multiuser detection in UWB systems, a combined scheme is proposed, which couples the minimum-mean-square-error and the DTI subspace tracking algorithm under UWB time-variant channels. Simulation results show that DTI is able to fast and precisely trace the variation of the channel environment and to improve the performance of the blind adaptive multiuser detection with a subspace approach over time-varying channels.
\end{abstract}

Keywords Blind multiuser detection · Multiple access interference ·

Decision timing instant · Minimum-mean-square-error · Ultra-wideband system

\section{Introduction}

Ultra-wideband (UWB) technology is currently regarded as an attractive solution for many wireless applications where high resolution, reduced interference, and propagation around obstacles are challenging [1-3]. Also, the research of UWB systems has recently attracted a significant interest in both the academic and industrial community [1-3]. It utilizes ultrashort pulse shapes transmitted at a very low power spectral density (PSD) in compliance

H.-L. Hung $(\varangle)$

Department of Electrical Engineering, Chien-Kuo Technology University, No.1 Chien Shou Rd., Changhua, Taiwan

e-mail: hlh@ctu.edu.tw

C.-H. Cheng

Department of Electrical Engineering, National Formosa University, Yunlin, Taiwan

e-mail: chcheng@nfu.edu.tw 
to the Federal Communications Commission (FCC) rules that defined UWB signals with a fractional bandwidth greater than or equal to 0.2 of the center frequency or a bandwidth of at least $500 \mathrm{MHz}$ [4]. In recent years, much research work has been devoted to accelerating the acquisition process of UWB signals. Based on different algorithmic approaches, several fast acquisition techniques were proposed [5-10]. However, the complexity aspect was generally less emphasized than the algorithmic one. Indeed, the correlations are computed in the time domain and acquisition systems are fed by stream processing, sample by sample, irrespective of the search strategy [4]. The corresponding architectures are thus not optimal and may require relatively long processing times under challenging conditions, e.g., inside an underground mine gallery, a peculiar, harsh and infrequently studied environment $[1,11]$ where line-of- sight (LOS) ranging applications are highly desirable for safety and efficiency purposes. However, UWB technology, as a physical support with excellent temporal resolution, is very promising for such applications [12]. With on-going widespread deployment of the UWB communication systems, reliable signal detection is desirable. Most existing approaches employ correlators to correlate the received signal with a template signal [13]. This technique appears very powerful, but not so satisfactory in a multipath and multiple access channels. Therefore, both of the unknown multiple access interference (MAI) and the multipath distortion need to be mitigated.

Recent works have focused on obtaining low-complexity algorithms for rapid timing acquisition by making use of coarse bin search and exploiting coded beacon sequences in conjunction with a correlator bank or subspace-based spectral estimation [14,15]. Reference $[16,17]$ proposes a semi-blind synchronization scheme based on maximum-likelihood (ML) techniques to recover symbol and frame timing. Semi-blind schemes combine the methods of channel and timing acquisition based on pilot symbols and blind channel and timing acquisition derived directly from the data conveying signal. However, each of these approaches requires one or more of the following assumptions: multipath is absent; time-hopping (TH) codes are slow or even absent; the multipath channel is known; and the system can afford the prohibitive complexity of exhaustively searching over thousands of bins (chips). Evidently, timing algorithms based on these assumptions are impractical for most realistic UWB settings. In addition, the estimation of the time-of arrival (TOA) is a particular case of a timing acquisition problem whose maximum likelihood (ML) solution is known [18] but has strong practical limitations due to the requirement of very high sampling rates and complexity. Although there are different ML approaches that manage to reduce complexity considerably [19], there still exist practical limitations for their use in positioning applications. Efforts have been steered towards less complex solutions, yet aiming at near optimum performance. Most of them are based on time domain approaches. Under the assumption of known undistorted received pulses, optimal correlation-based TOA estimators [20] and a simplified version of the generalized ML criterion [13] are known. Also, energy based TOA estimation schemes have received considerable attention as a viable alternative to correlation-based methods [21,22] due to their reduced complexity implementation at sub-Nyquist sampling rates. Indeed, they do not require expensive pulse-shape estimation algorithms and represent a good solution for low power and low-complexity systems at the expense of ranging accuracy. Hence, In order to achieve a low-complexity receiver, an UWB computationally-efficient acquisition system showing explicit design characteristics that offer greatly improved computational cost and acquisition time was proposed in this paper.

In this paper, we evaluate the performance of this new UWB fast acquisition system suggested for ranging over this peculiar time-varying channel. For the TH-UWB systems, the main focus of our work is on the study of the miltiuser detection. In the multiuser transmission environment, MAI becomes a serious issue and results in the degradation of the system 
performance. To efficiently suppress MAI, a subspace-based blind adaptive linear detector which was first proposed by Poor [23] is chosen. Besides, since the UWB systems are mainly operated in an indoor environment, it also causes serious inter-symbol interference (ISI). Moreover, we consider a mobile UWB transceiver. Changing of the transmission position will sometimes suffer the transition of the channel models because of different transmission distance or characteristics, for example, from CM1 to CM4. However, a subspace-based blind adaptive linear detector proposed by H. V. Poor is highly sensitive to the sudden change of the channel environment over a time-varying channel, eventually results in large performance degradation and a slow convergence speed to steady state, as illustrated in simulation results. It is because the subspace tacking algorithm is not update the signal subspace components rapidly under the changing environment. In order to overcome this shortcoming, we propose a low complexity decision mechanism, termed the decision timing instant (DTI) [24]. The proposed DTI method is mainly designed to accurately detect the change of the channel environment over a time-varying channel. In following sections, we will introduce the design theorem of the DTI method, and then the combination of DTI method and subspace-based blind adaptive linear detector for TH-UWB systems is investigated as well. The remainder of this paper is organized as follows. In Sect. 2, the system model is given. Section 3 details the proposed UWB fast acquisition system with its implementation issues, followed by Sect. 4 that provides simulation results, and finally Sect. 5 concludes the paper.

\section{UWB Multipath Channel Model}

The overall system model is shown in Fig. 1. For the evaluation of the performance, we require an accurate model for the UWB propagation channel. The IEEE 802.15.3a standard UWB channel model which is based on a number of indoor channel measurements in the 2-8 GHz band and modified from the traditional Saleh-Valenzuela model [25] is considered here. This model is similar to the Saleh-Valenzuela (S-V) model with the channel coefficients that is log-normal distribution instead of Rayleigh distribution. In addition, the independent fading is assumed for each cluster as well as each ray within the cluster. We assume that the propagation channel is modeled by the UWB indoor channel model described in [26]:

$$
h(t)=\sum_{j=0}^{J} \sum_{q=0}^{Q} \alpha_{q, j} \delta\left(t-T_{j}-\tau_{q, j}\right)=\sum_{l=1}^{L} \tilde{h}_{l} \delta\left(t-\tau_{l}\right)
$$

where $\left\{\alpha_{q, j}\right\}$ is the multipath gain coefficient, $\left\{T_{j}\right\}$ is the delay of the $j$ th cluster, $\left\{\tau_{q, j}\right\}$ is the delay of the $q$ th multipath component (ray) relative to the $j$ th cluster arrival time $\left(T_{j}\right)$. $\tilde{h}_{l}$ is the gain coefficient of the $l$ th multipath, and $\tau_{l}$ is the delay of the $l$ th multipath. By definition, we have $\tau_{0, j}=0$. The distribution of the cluster arrival time and the ray arrival time are given by

$$
\begin{aligned}
p\left(T_{j} \mid T_{j-1}\right) & =\Lambda \exp \left[-\Lambda\left(T_{j}-T_{j-1}\right)\right], j>0 \\
p\left(\tau_{q, j} \mid \tau_{(q-1), j}\right) & =\lambda \exp \left[-\lambda\left(\tau_{q, j}-\tau_{(q-1), j}\right)\right], q>0
\end{aligned}
$$

where $\Lambda$ is the cluster arrival rate and $\lambda$ is the ray arrival rate. The channel coefficients are defined as follows $[27,28]$ :

$$
\alpha_{q, j}=p_{q, j} \beta_{q, j}^{\prime}
$$

where $p_{q, j}$ is a random equi-probable \pm 1 and $\beta_{q, j}^{\prime}$ is the log-normal distributed fading component, i.e. $20 \log _{10}\left(\beta_{q, j}^{\prime}\right) \propto \operatorname{Normal}\left(\mu_{q, j}, \sigma^{2}\right)$. The expectation value, $\mu_{q, j}$, is 


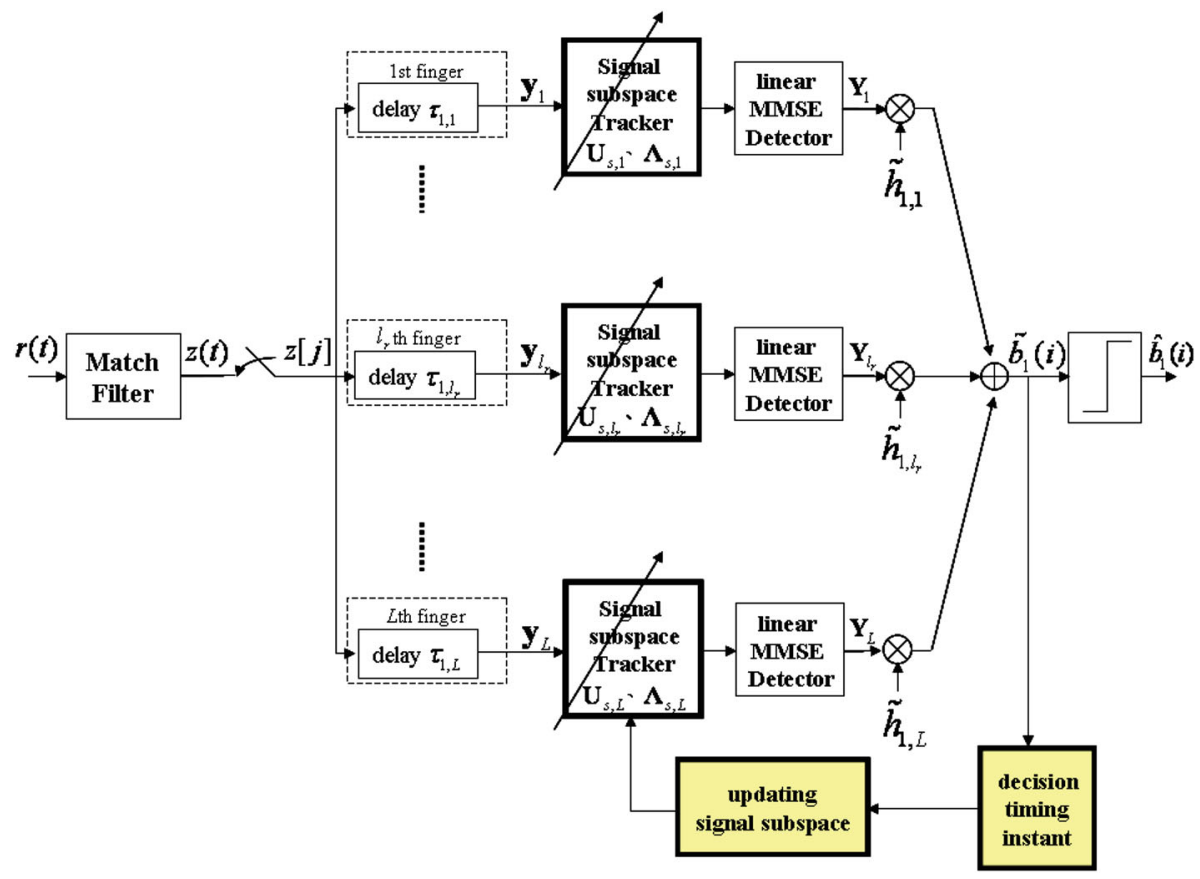

Fig. 1 The receiver structure for decision timing instant

$$
\mu_{q, j}=\frac{10 \ln \left(\Omega_{0}\right)-10 T_{j} / \Gamma-10 \tau_{q, j} / \gamma}{\ln (10)}-\frac{\sigma^{2} \ln (10)}{20}
$$

where $\Omega_{0}$ is the mean energy of the first path of the first cluster. $\Gamma$ and $\gamma$ are the cluster decay factor and ray decay factor, respectively. The expectation of $\beta_{q, j}^{\prime 2}$ is defined as the decaying multipath intensity profile,

$$
E\left[\beta_{q, j}^{\prime 2}\right]=\Omega_{0} e^{-T_{j} / \Gamma} e^{-\tau_{q, j} / \gamma},
$$

There are four different kinds of cases of the modified S-V model that correspond with different channel conditions as described in Table $1 . \mathrm{NP}_{10 \mathrm{~dB}}$ is the number of paths within $10 \mathrm{~dB}$ of the strongest path. NP $(85 \%)$ gives the number of paths containing $85 \%$ of the total multipath energy. The channel coefficients are normalized as $\sum_{l=1}^{L} \tilde{h}_{l}^{2}=1$ in order to remove the path loss factor from consideration.

UWB radios are seen as promising candidates for adoption for high data rate communications, mainly due to their large transmission bandwidth, defined by the FCC to be either at least $500 \mathrm{MHz}$, or more than $20 \%$ of their center frequency. We consider a binary phase-shift keyed (BPSK) random time-hopping impulse-radio (TH-IR) system where the transmitted signal from user $k$ in a $K$-user setting is represented by the following general model:

$$
s_{t x}^{(k)}(t)=A_{k} \sum_{n=-\infty}^{\infty} b_{k}(n) \sum_{m=0}^{N_{f}-1} d_{k}(m) w\left(t-n T_{s}-m T_{f}-c_{m}^{(k)} T_{c}\right)
$$

where $w(t)$ is the transmitted UWB pulse with unit energy, which is usually referred to as the monocycle. $A_{k}$ is the amplitude of user $k, T_{f}$ is the average pulse repetition time(also called 
Table 1 The IEEE UWB Channel Characteristic [25]

\begin{tabular}{|c|c|c|c|c|}
\hline Target channel Characteristics & $\mathrm{CM} 1^{\mathrm{a}}$ & $\mathrm{CM} 2^{\mathrm{b}}$ & $\mathrm{CM} 3^{\mathrm{c}}$ & $\mathrm{CM} 4^{\mathrm{d}}$ \\
\hline Distance (m) & $0-4$ & $0-4$ & $4-10$ & \\
\hline (Non-) Line-of-sight & LOS & NLOS & NLOS & NLOS \\
\hline Mean excess delay $(\mathrm{ns})\left(\tau_{m}\right)$ & 5.05 & 10.38 & 14.18 & \\
\hline RMS delay $(\mathrm{ns})\left(\tau_{r m s}\right)$ & 5.28 & 8.03 & 14.28 & 25 \\
\hline $\mathrm{NP}_{10 \mathrm{~dB}}$ & & & 35 & \\
\hline $\mathrm{NP}(85 \%)$ & 24 & 36.1 & 61.54 & 122.8 \\
\hline
\end{tabular}

a This model is based on LOS $(0-4 \mathrm{~m})$ channel measurements

b This model is based on NLOS $(0-4 \mathrm{~m})$ channel measurements

c This model is based on NLOS $(4-10 \mathrm{~m})$ channel measurements and NLOS measurements

$\mathrm{d}$ This model is generated to fit a $25 \mathrm{nsec}$ RMS delay spread to represent an extreme NLOS multipath channel

the "frame" time), $N_{f}$ is the number of pulses representing one information symbol of length $T_{s}$, and $b_{k} \in\{+1,-1\}$ is the information symbol transmitted by user $k$. In order to allow the channel to be exploited by many users and avoid catastrophic collisions, a time-hopping (TH) sequence $\left\{c_{m}^{(k)}\right\}$ is assigned to each user, where $c_{m}^{(k)} \in\left\{0,1, \ldots, N_{c}-1\right\}$ with equal probability, and $c_{m}^{(k)}$ and $c_{p}^{(q)}$ are independent for $(k, m) \neq(q, p)$. This TH sequence provides an additional time shift of $c_{m}^{(k)} T_{c}$ seconds to the $m$ th pulse of the $k$ th user where $T_{c}$ is the chip time and is chosen to satisfy $T_{c} \leq T_{f} / N_{c}$ in order to avoid interpulse interference (IPI). Without loss of generality, $T_{f}=N_{c} T_{c}$ is assumed throughout the paper. Random polarity codes $d_{k}(m)$ are binary random variables taking values $\{ \pm 1\}$ with equal probability and $d_{k}(m)$ and $d_{q}(p)$ are independent for $(k, m) \neq(q, p)$. With a modification of the definition proposed by Fishler and Poor [29], we redefine a sequence $\left\{s_{k}(m)\right\}$ as follows

$$
s_{k}(m)= \begin{cases}d_{k}\left(\left\lfloor m / N_{c}\right\rfloor\right), & m-N_{c}\left(\left\lfloor m / N_{c}\right\rfloor\right)=c_{\left\lfloor m / N_{c}\right\rfloor}^{(k)} \\ 0, & \text { otherwise }\end{cases}
$$

where $\lfloor\cdot\rfloor$ denotes the integer part.

The sequence $\left\{s_{k}(m)\right\}$ can be regarded as a chip rate spreading sequence where $s_{k}(m)$ take the value $d_{k}\left(\left\lfloor m / N_{c}\right\rfloor\right)$ whenever a pulse is transmitted and zero otherwise. The transmitted signal can be rewritten by the following model:

$$
s_{t x}^{(k)}(t)=A_{k} \sum_{n=-\infty}^{\infty} b_{k}(n) \sum_{m=0}^{N-1} s_{k}(m) w\left(t-n T_{s}-m T_{c}\right)
$$

where $N \equiv N_{f} N_{c}$

Propagation over an indoor channel has the main effect of introducing multiple delayed and attenuated replicas of a transmitted pulse, corresponding to the different propagation paths between transmitter and receiver. To simplify analysis, multipath arrivals are assumed to be at integral multiples of the minimum resolution time, $T_{c}$. The impulse response for user $k$ :

$$
h_{k}(t)=\sum_{l=1}^{L_{k}} \tilde{h}_{k, l} \delta\left(t-(l-1) T_{c}\right)
$$

where $\tilde{h}_{k, l}$ and $L_{k}$ are the channel coefficient of the $l$ th path and the number of paths for user $k . \delta(\cdot)$ is the Dirac delta function. $T_{c}$ is the minimum resolvable path interval. 
For the purpose of analysis, it is assumed throughout the rest of the thesis that the system is a synchronous system. The received signal $r(t)$ can be expressed as follows:

$$
r(t)=\sum_{k=1}^{K} s_{r x}^{(k)}(t)+v(t)
$$

where $v(t)$ is a zero mean white Gaussian noise process with two-sided spectral density $N_{0} / 2$ and $s_{r x}^{(k)}(t)$, the received continuous signal from the $k$ th user for the UWB system, can be expressed by

$$
\begin{aligned}
s_{r x}^{(k)}(t) & =s_{t x}^{(k)}(t) * h_{k}(t) \\
& =A_{k} \sum_{n=-\infty}^{\infty} b_{k}(n) \sum_{m=0}^{N-1} s_{k}(m)\left[w\left(t-n T_{s}-m T_{c}\right) * h_{k}(t)\right] \\
& =A_{k} \sum_{n=-\infty}^{\infty} b_{k}(n) \sum_{m=0}^{N-1} s_{k}(m) \sum_{l=1}^{L_{k}} \tilde{h}_{k, l} w\left(t-n T_{s}-m T_{c}-(l-1) T_{c}\right)
\end{aligned}
$$

where $*$ denotes convolution.

Instead of first reconstructing the received signal, the received signal $r(t)$ of Eq. (10) is passed through a linear filter matched to the received pulse $w(t)$, and the output of this filter is sampled every $T_{C}$ seconds. The output of chip matched filter is

$$
\begin{aligned}
z(t) & =r(t) * w(-t) \\
& =\sum_{k=1}^{K} A_{k} \sum_{n=-\infty}^{\infty} b_{k}(n) \sum_{m=0}^{N-1} s_{k}(m) \sum_{l=1}^{L_{k}} \tilde{h}_{k, l} w\left(t-n T_{s}-m T_{c}-(l-1) T_{c}\right) * w(-t)+v(t) * w(-t) \\
& =\sum_{k=1}^{K} A_{k} \sum_{n=-\infty}^{\infty} b_{k}(n) \sum_{m=0}^{N-1} s_{k}(m) \sum_{l=1}^{L_{k}} \tilde{h}_{k, l} \delta\left(t-n T_{s}-m T_{c}-(l-1) T_{c}\right)+\tilde{v}(t)
\end{aligned}
$$

Then, the sample output of the receiver at the $j$ th chip of the $i$ th bit is represented as

$$
\begin{aligned}
z[j] & =\left.z(t)\right|_{t=i T_{s}+j T_{c}} \\
& =\sum_{k=1}^{K} A_{k} \sum_{n=-\infty}^{\infty} b_{k}(n) \sum_{m=0}^{N-1} s_{k}(m) \sum_{l=1}^{L_{k}} \tilde{h}_{k, l} \delta\left((i-n) T_{s}+(j-m) T_{c}-(l-1) T_{c}\right)+\tilde{v}[j]
\end{aligned}
$$

The $N$-vector of chip-matched filter output during a symbol duration:

$$
\begin{aligned}
\mathbf{Z}[i] & =\left[z[1] z[2] \ldots z[N]^{T}\right. \\
& =\sum_{k=1}^{K} A_{k} \sum_{n=-\infty}^{\infty} b_{k}(n) \sum_{l=1}^{L_{k}} \tilde{h}_{k, l} \mathbf{s}_{k, l}+\mathbf{v}^{\prime}[\mathbf{i}]
\end{aligned}
$$

where $\mathbf{s}_{k, l}$ is the vector representation of the delayed user signature waveform $s_{k}\left(t-(l-1) T_{c}\right)$. $\mathbf{v}^{\prime}[i]=[\tilde{v}[1] \tilde{v}[2] \ldots \tilde{v}[N]]^{T}(N \times 1$ vector $)$ is the noise vector corresponding to the $i$ th bit. The vector form of the $l_{r}$ th finger of the Rake receiver is

$$
\mathbf{y}_{l_{r}}[i]=\left[z\left[l_{r}\right] z\left[l_{r}+1\right] \ldots z\left[N+l_{r}-1\right]\right]_{(N \times 1)}^{T}
$$

In this paper, we assume that the maximum delay spread is within one symbol duration. Hence, for the $l_{r}$ th finger, Eq. (15) can be reformulated as the following matrix form: 


$$
\mathbf{y}_{l_{r}}[i]=A_{1} b_{1}(i) \tilde{\mathbf{s}}_{1} \mathbf{h}_{1}+I S I+M A I+A W G N
$$

where

$$
\begin{aligned}
& I S I=A_{1} b_{1}(i-1) \tilde{\mathbf{s}}_{1}^{-} \mathbf{h}_{1}+A_{1} b_{1}(i+1) \tilde{\mathbf{s}}_{1}^{+} \mathbf{h}_{1} \\
& M A I=\sum_{k=2}^{K} A_{k} b_{k}(i-1) \tilde{\mathbf{s}}_{k}^{-} \mathbf{h}_{k}+A_{k} b_{k}(i) \tilde{\mathbf{s}}_{k} \mathbf{h}_{k}+A_{k} b_{k}(i+1) \tilde{\mathbf{s}}_{k}^{+} \mathbf{h}_{k} \\
& A W G N=\mathbf{v}[i]
\end{aligned}
$$

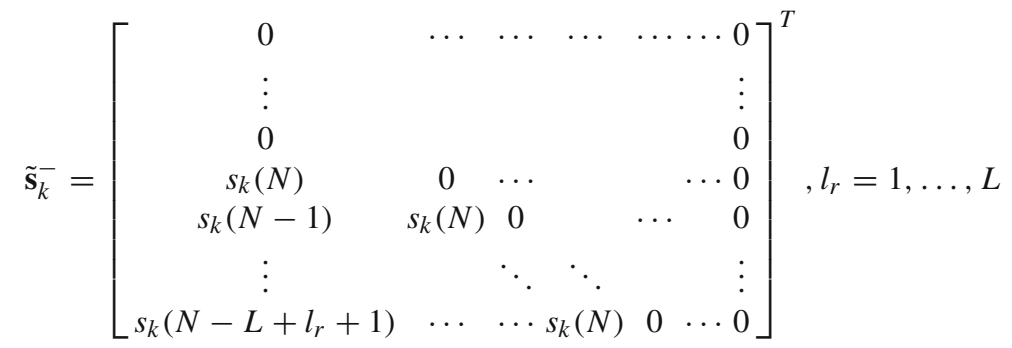

$\left(N \times L\right.$ matrix), $L$ is the maximum of $L_{k}, k=1, \ldots, K$,

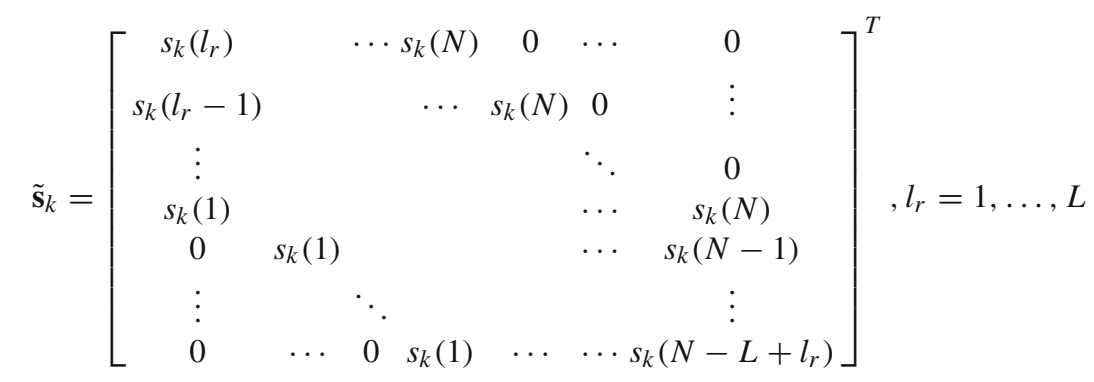

$(N \times L$ matrix $)$ and

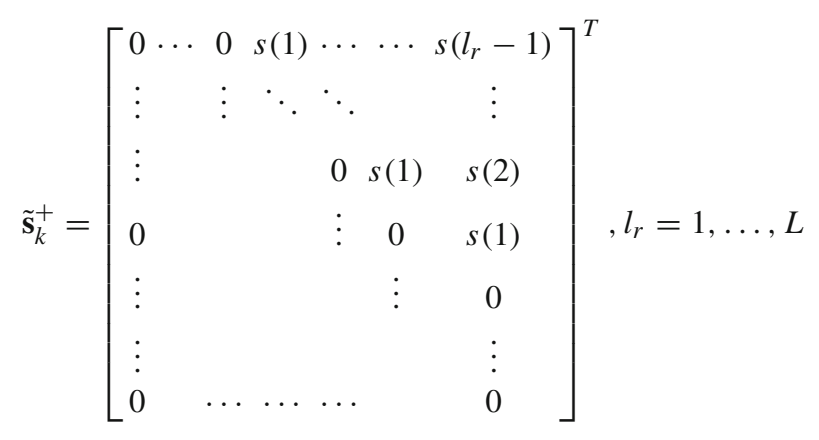

$(N \times L$ matrix $)$ are the signature.

$\mathbf{h}_{k}=\left[\tilde{h}_{k, 1} \ldots \tilde{h}_{k, L_{k}}\right](L \times 1$ vector $)$ is the vector of channel coefficient for $k$ th user.

$\mathbf{v}[i]=\left[\tilde{v}\left[l_{r}\right] \tilde{v}[2] \ldots \tilde{v}\left[N+l_{r}-1\right]\right]^{T}(N \times 1$ vector $)$

In Eq. (16), the first term contains the $i$ th dit of the user 1 ; the second term contains the ISI from the $(i-1)$ th and $(i+1)$ th bits of the user 1 ; the third term contains the MAI from other users, and the last term is the ambient channel noise. We can rewrite $\mathbf{y}_{l_{r}}$. $\left.i\right]$ in a compact form as 


$$
\begin{aligned}
\mathbf{y}_{l_{r}}[i] & =\underbrace{\tilde{\mathbf{S}}^{-} \mathbf{H A}}_{\tilde{\mathbf{G}}_{l_{r}}^{-}} \mathbf{b}(i-1)+\underbrace{\tilde{\mathbf{S}} \mathbf{H} \mathbf{A}}_{\tilde{\mathbf{G}}_{l_{r}}} \mathbf{b}(i)+\underbrace{\tilde{\mathbf{S}}^{+} \mathbf{H A}}_{\tilde{\mathbf{G}}_{l_{r}}^{+}} \mathbf{b}(i+1)+\mathbf{v}[i] \\
& =\left[\begin{array}{lll}
\tilde{\mathbf{G}}_{l_{r}}^{-} & \tilde{\mathbf{G}}_{l_{r}} & \tilde{\mathbf{G}}_{l_{r}}^{+}
\end{array}\right]\left[\begin{array}{c}
\mathbf{b}(i-1) \\
\mathbf{b}(i) \\
\mathbf{b}(i+1)
\end{array}\right]+\mathbf{v}[i]=\mathbf{G}_{l_{r}} \mathbf{B}+\mathbf{v}[i]
\end{aligned}
$$

where $\tilde{\mathbf{S}}^{-}=\left[\tilde{\mathbf{s}}_{1}^{-} \ldots \tilde{\mathbf{s}}_{K}^{-}\right](N \times K L$ matrix $), \tilde{\mathbf{S}}=\left[\tilde{\mathbf{s}}_{1} \ldots \tilde{\mathbf{s}}_{K}\right](N \times K L$ matrix $)$, $\tilde{\mathbf{S}}^{+}=\left[\tilde{\mathbf{s}}_{1}^{+} \ldots \tilde{\mathbf{s}}_{K}^{+}\right](N \times K L$ matrix $), \mathbf{H}=\operatorname{diag}\left\{\mathbf{h}_{1} \ldots \mathbf{h}_{K}\right\}(K L \times K$ matrix $), \mathbf{A}=$ $\operatorname{diag}\left\{A_{1} \ldots A_{K}\right\}(K \times K$ matrix $), \mathbf{b}(i)=\left[b_{1}(i) \ldots b_{K}(i)\right]^{T}(K \times 1$ matrix $), \mathbf{G}_{l_{r}}=$ $\left[\tilde{\mathbf{G}}_{l_{r}}^{-} \tilde{\mathbf{G}}_{l_{r}} \tilde{\mathbf{G}}_{l_{r}}^{+}\right](N \times 3 K$ matrix $)$, and $\mathbf{B}=[\mathbf{b}(i-1) \mathbf{b}(i) \mathbf{b}(i+1)]^{T}(3 K \times 1$ matrix $)$. The output of $l_{r}$ th finger is passed through a linear multiuser detector $\mathbf{w}_{1, l_{r}}$ for the 1 th user's data, we have

$$
\mathbf{Y}_{l_{r}}[i]=\mathbf{w}_{1, l_{r}}^{T} \mathbf{y}_{l_{r}}[i]
$$

The $L$ finger outputs $\left\{\mathbf{Y}_{l_{r}}[i]\right\}$ 's are individually weighted and then combined by a linear combiner to form the 1 th user decision variable given by

$$
\tilde{b}_{1}(i)=\sum_{l_{r}=1}^{L} \tilde{h}_{1, l_{r}} \mathbf{Y}_{l_{r}}[i]
$$

where $\tilde{h}_{1, l_{r}}$ is the weight of the $l_{r}$ th finger for the user 1 . Then, the output of the decision device of desired user is

$$
\hat{b}_{1}(i)=\operatorname{sgn}\left(\tilde{b}_{1}(i)\right)
$$

\section{Subspace Concept for UWB Systems}

\subsection{Subspace Concept}

We assume that the additive noise is white and independent of the user signal. From Eq. (17), the $N \times N$ autocorrelation matrix which is obtained from the $l_{r}$ th finger of the Rake receiver can be denoted as:

$$
\mathbf{C}_{l_{r}}=\mathrm{E}\left\{\mathbf{y}_{l_{r}} \mathbf{y}_{l_{r}}^{T}\right\}=\mathbf{G}_{l_{r}} \mathbf{G}_{l_{r}}^{T}+\sigma^{2} \mathbf{I}_{N}
$$

where $E[\cdot]$ denotes the mathematical expectation. Let $\lambda_{j}$ and $\mathbf{u}_{j}(j=1,2, \ldots, N)$ be the eigenvalues and corresponding eigenvectors of $\mathbf{C}_{l_{r}}$. Obviously, it is reasonable to assume that $\mathbf{u}_{j}(j=1,2, \ldots, N)$ are linearly independent and all the eigenvalues have following property [31]

$$
\begin{aligned}
& \lambda_{j}>\sigma^{2} \text { for } j=1, \ldots, K \\
& \lambda_{j}=\sigma^{2} \text { for } j=K+1, \ldots, N
\end{aligned}
$$

The dominant eigenpairs $\left(\lambda_{j}, \mathbf{u}_{j}\right)$ for $j=1, \ldots, K$ are termed as the signal eigenvalues and signal eigenvectors while $\left(\lambda_{j}, \mathbf{u}_{j}\right)$ for $j=K+1, \ldots, N$ are referred to as the noise eigenvalues and noise eigenvectors. Let

$$
\mathbf{U}_{s, l_{r}}=\left[\mathbf{u}_{1} \ldots \mathbf{u}_{K}\right]
$$

and

$$
\mathbf{U}_{n, l_{r}}=\left[\mathbf{u}_{K+1} \ldots \mathbf{u}_{N}\right],
$$


which are defined as the signal and noise subspace, respectively. Since $\mathbf{C}_{l_{r}}$ is symmetric and nonnegative definite, we have

$$
\mathbf{C}_{l_{r}}=\mathbf{U}_{l_{r}} \boldsymbol{\Lambda}_{l_{r}} \mathbf{U}_{l_{r}}^{-1}=\left[\mathbf{U}_{s, l_{r}} \mathbf{U}_{n, l_{r}}\right]\left[\begin{array}{cc}
\boldsymbol{\Lambda}_{s, l_{r}} & \\
& \boldsymbol{\Lambda}_{n, l_{r}}
\end{array}\right]\left[\mathbf{U}_{s, l_{r}} \mathbf{U}_{n, l_{r}}\right]^{-1},
$$

where $\mathbf{U}_{l_{r}}=\left[\mathbf{U}_{s, l_{r}} \mathbf{U}_{n, l_{r}}\right]$, and $\boldsymbol{\Lambda}_{l_{r}}=\operatorname{diag}\left(\boldsymbol{\Lambda}_{\mathrm{s}, l_{r}}, \boldsymbol{\Lambda}_{n, l_{r}}\right) ; \boldsymbol{\Lambda}_{s, l_{r}}=\operatorname{diag}\left(\boldsymbol{\lambda}_{1}, \ldots, \lambda_{K}\right)$ and $\boldsymbol{\Lambda}_{n, l_{r}}$ $=\operatorname{diag}\left(\sigma^{2}, \ldots, \sigma^{2}\right)$. Here, $\mathbf{U}_{s, l_{r}}$ and $\mathbf{U}_{n, l_{r}}$ are only linear independent. It should be noted that if the column vectors of $\mathbf{U}_{l_{r}}$ are orthonormal eigenvectors, we have $\mathbf{U}_{l_{r}}^{T}=\mathbf{U}_{l_{r}}^{-1}$; thus, we get

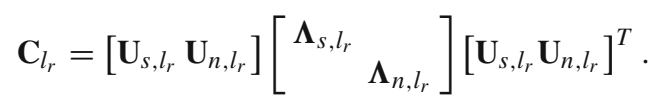

It is easy to verify that $\mathbf{U}_{s, l_{r}}$ and $\mathbf{S}$ have the same column span, i.e., $\operatorname{range}\left(\mathbf{U}_{s, l_{r}}\right)=\operatorname{range}(\mathbf{S})$, and its orthogonal complement, the noise subspace, is spanned by the column vectors of $\mathbf{U}_{n, l_{r}}$ [30,31]. Define the $N \times N$ diagonal matrix

$$
\Lambda_{0, l_{r}} \triangleq \Lambda_{l_{r}}-\sigma^{2} \mathbf{I}_{N}=\operatorname{diag}\left(\lambda_{1}-\sigma^{2}, \ldots, \lambda_{k}-\sigma^{2}, 0 \ldots, 0\right)
$$

form Eqs. (21) to (22) we get

$$
\mathbf{G}_{l_{r}} \mathbf{G}_{l_{r}}^{T}=\mathbf{U}_{s, l_{r}}\left(\boldsymbol{\Lambda}_{s, l_{r}}-\sigma^{2} \mathbf{I}_{\mathbf{K}}\right) \mathbf{U}_{s, l_{r}}^{T}=\mathbf{U}_{l_{r}} \boldsymbol{\Lambda}_{0, l_{r}} \mathbf{U}_{l_{r}}^{T}
$$

\subsection{Subspace-Based Linear Multiuser Detectors}

Suppose that we are interested in demodulating the data bits of the $l_{r}$ th finger of user 1 . We use $\mathbf{d}_{1, l_{r}}$ and $\mathbf{m}_{1, l_{r}}$ represent the weight vector of the decorrelating detector and the minimum-mean-square-error (MMSE) detector, respectively. The linear decorrelating detector for detecting the $i$ th bit of the user 1 has the form of Eq. (21) with the weight vector $\mathbf{w}_{1, l_{r}}=\mathbf{d}_{1, l_{r}}$ such that, in the absence of noise, both the MAI and the ISI are completely eliminated.

Proposition 1 A linear decorrelating detector for detecting the 1th user's data bit $b_{1}(i)$ from the output signal of $l_{r}$ th finger is given by [32]

$$
\mathbf{d}_{1, l_{r}}=\mathbf{U}_{s, l_{r}}\left(\boldsymbol{\Lambda}_{s, l_{r}}-\sigma^{2} \mathbf{I}_{K}\right)^{-1} \mathbf{U}_{s, l_{r}}^{T} \mathbf{g}_{1}
$$

where $\mathbf{g}_{1}=\mathbf{G}_{l_{r}} 1_{K+1}$ and $1_{K+1}$ is a $3 K$-vector with all entries zeros except for the $K+1$ th entry.

Proposition 2 A linear MMSE detector for detecting the 1th user's data bit $b_{1}(i)$ from the output signal of $l_{r}$ th finger is given by [32]

$$
\mathbf{m}_{1, l_{r}}=\mathbf{U}_{s, l_{r}} \boldsymbol{\Lambda}_{s, l_{r}}^{-1} \mathbf{U}_{s, l_{r}}^{T} \mathbf{g}_{1}
$$

Since the signal subspace components $\left(\mathbf{U}_{s, l_{r}}, \boldsymbol{\Lambda}_{\mathbf{s}, l_{r}}\right.$, and $\left.\sigma^{2}\right)$, as well as the composite signature waveform of the desired user, $\mathbf{g}_{1}$, can be estimated from the received signal, with the prior knowledge of only the spreading sequence of the desired user.

\subsection{Tracking the Signal Subspace}

It is seen from the previous section that the linear multiuser detectors are obtained once the signal subspace components are identified. The classic approach to subspace estimation is through batch eigenvalue decomposition (ED) of the sample autocorrelation matrix, or 
batch singular value decomposition (SVD) of the data matrix, which is computationally too expensive for adaptive applications. Modern subspace tracking algorithms are recursive in nature and update the subspace in a sample-by-sample fashion [31,34]. In this paper, we adopt the proposed projection approximation subspace tracking (PASTd) algorithm [30,33] for the blind adaptive multiuser detection application. The advantages of this algorithm include almost sure global convergence to the signal eigenvectors and eigenvalues, low computational complexity $(O(K))$. We next briefly review the PASTd algorithm for tracking the signal subspace. Let $\mathbf{y}_{l_{r}} \in \Re^{N}$ be a random vector with autocorrelation matrix $\mathbf{C}_{l_{r}}=\mathrm{E}\left\{\mathbf{y}_{l_{r}} \mathbf{y}_{l_{r}}^{T}\right\}$. Consider the scalar function

$$
\begin{aligned}
\mathbf{J}(\mathbf{W}) & =E\left\{\left\|\mathbf{y}_{l_{r}}-\mathbf{W} \mathbf{W}^{T} \mathbf{y}_{l_{r}}\right\|^{2}\right\} \\
& =\operatorname{tr}\left(\mathbf{C}_{l_{r}}\right)-2 \operatorname{tr}\left(\mathbf{W}^{T} \mathbf{C}_{l_{r}} \mathbf{W}\right)+\operatorname{tr}\left(\mathbf{W}^{T} \mathbf{C}_{l_{r}} \mathbf{W} \mathbf{W}^{T} \mathbf{W}\right)
\end{aligned}
$$

with a matrix argument $\mathbf{W} \in \mathfrak{R}^{N \times K}(K<N)$. It is shown in [31] that

$\mathbf{W}$ is a stationary point of $J(\mathbf{W})$ if and only if $\mathbf{W}=\mathbf{U}_{K, l_{r}} \mathbf{Q}$, where $U_{K, l_{r}} \in \mathfrak{R}^{N \times K}$ contains any $K$ distinct eigenvectors of $\mathbf{C}_{l_{r}}$ and $\mathbf{Q} \in \mathfrak{R}^{K \times K}$ is any unitary matrix.

All stationary points of $J(\mathbf{W})$ are saddle points except when $\mathbf{U}_{K, l_{r}}$ contains the $K$ dominant eigenvectors of $\mathbf{C}_{l_{r}}$. In that case, $J(\mathbf{W})$ attains the global minimum. Therefore, for $K=1$, the solution of minimizing $J(\mathbf{W})$ is given by the most dominant eigenvector of $\mathbf{C}_{l_{r}}$. In applications, only sample vectors $\mathbf{y}_{l_{r}}(n)$ are available. Replacing Eq. (30) with the exponentially weighted sums yields

$$
\mathrm{J}[\mathbf{W}(t)]=\sum_{n=1}^{t} \beta^{t-n}\left\|\mathbf{y}_{l_{r}}(n)-\mathbf{W}(t) \mathbf{W}(t)^{T} \mathbf{y}_{l_{r}}(n)\right\|^{2}
$$

The key issue of the PASTd approach is to approximate $\mathbf{W}(t)^{T} \mathbf{y}_{l_{r}}(n)$ in Eq. (28), the unknown projection of $\mathbf{y}_{l_{r}}(n)$ onto the columns of $\mathbf{W}(t)$, by $\tilde{\mathbf{y}}(n)=\mathbf{W}(n-1)^{T} \mathbf{y}_{l_{r}}(n)$, which can be calculated for $1 \leq n \leq t$ at time $t$. This results in a modified cost function

$$
\tilde{\mathbf{J}}[\mathbf{W}(t)]=\sum_{n=1}^{t} \beta^{t-n}\left\|\mathbf{y}_{l_{r}}(n)-\mathbf{W}(t) \tilde{\mathbf{y}}(n)\right\|^{2}
$$

The recursive least squares (RLS) algorithm can then be used to solve for $\mathbf{W}(t)$ that minimizes the exponentially weighted least squares criterion Eq. (32). The signal-to-noise ratio before spreading is $20 \mathrm{~dB}$. The performance measure is the output signal-to-interference (SIR), defined as [34]

$$
\mathbf{S I R} \triangleq \frac{E^{2}\left\{\mathbf{w}_{1, l_{r}}^{T} \mathbf{y}_{l_{r}}\right\}}{\operatorname{Var}\left\{\mathbf{w}_{1, l_{r}}^{T} \mathbf{y}_{l_{r}}\right\}}=\frac{A_{1}^{2}\left(\mathbf{w}_{1, l_{r}}^{T} \mathbf{g}_{1}\right)^{2}}{\sum_{k=2}^{K} A_{k}^{2}\left(\mathbf{w}_{1, l_{r}}^{T} \mathbf{g}_{1}\right)^{2}+\sigma^{2}\left\|\mathbf{w}_{1, l_{r}}^{T}\right\|^{2}}
$$

\subsection{Design of Decision Timing Instant}

The design theorem adopts the statistical design and analysis [24]. The common statistics calculated are the mean and the variance. Here, we utilize the SIR iteratively to analyze the statistical property to calculate the mean and variance. The true values for the mean and variance are called population parameters, $\mu$ and $\sigma^{2}$. If the population parameters are unknown, we can use statistic to estimate the true mean and variance. So the point of taking a sample and computing a sample mean $M_{n}$ and a sample variance $V_{n}^{2}$ are to estimate the 
population mean and population variance, respectively. First of all, the SIR $X_{j}$ we calculate can be regarded as a random variable. Let $X_{1}, X_{2}, \ldots, X_{n}$ be a sequence of iid (independent identical distribution) random variables. The sample mean is

$$
M_{n}=\frac{1}{n} \sum_{j=1}^{n} X_{j}
$$

The sample variance is

$$
V_{n}^{2}=\frac{1}{n-1} \sum_{j=1}^{n}\left(X_{j}-M_{n}\right)^{2}
$$

Given any population with mean $\mu$ and variance $\sigma^{2}$, the sampling distribution of $M_{n}$ will be approximately normal with mean $M_{n}$ and variance $V_{n}^{2} / n$ when the sample size $n$ is large. The standard deviation of the distribution of the sample means is equal to $V_{n} / \sqrt{n}$ and is often called the standard error. This remarkable result is known as the central limit theorem. The theorem applies to any population with a finite standard deviation regardless of the shape of the underlying distribution. Using the standard error, we can calculate the likelihood that the true mean of the population is contained within an interval around the sample mean. This interval is called the confidence interval [24], and this may be expressed as a percentage or a decimal. Notice that a confidence interval for an unknown parameter provides both an estimate of the parameter and a measure of our confidence in that estimate. If $X$ is a random variable whose probability law depends on an unknown parameter, $\mu$. Given a random sample of $X$, call the sampled values $X_{1}, X_{2}, \ldots, X_{n}$, the two statistics $L_{1}$ and $L_{2}$ form a $100(1-\alpha) \%$ confidence interval for $\mu$ :

$$
P\left(L_{1} \leq \mu \leq L_{2}\right)=1-\alpha
$$

where the interval $\left[L_{1}, L_{2}\right]$ is confidence interval. The $1-\alpha$ is defined as the confidence level. We suppose that the pdf (probability density function) of Eq. (36) is normal distribution. We can represent the $100(1-\alpha) \%$ confidence interval for $\mu$ in a diagram as follows:

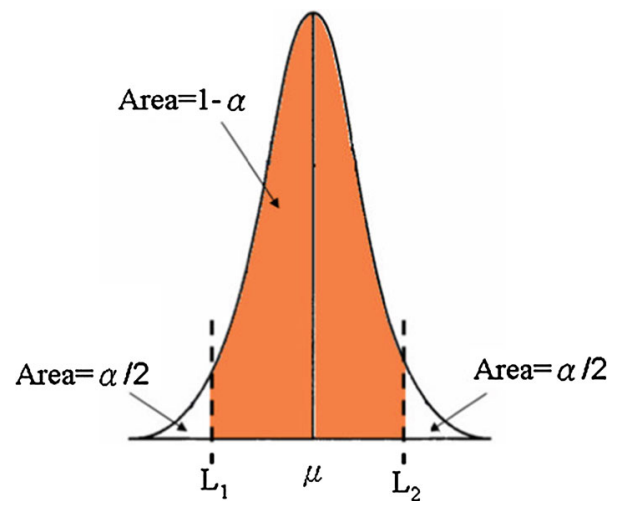

The orange (or shading) in the distribution indicates the confidence interval. Since the change of the environment decreases the performance, we only need to consider the lower bound in Eq. (36), that is to say, the $\mu$ of right side is confided. Therefore, we establish an interval by means of the concept of confidence interval to ensure that the environment is remain unchanged during the interval. In order to acquire the pdf of SIR, the statistic distribution in Fig. 12 is plotted. Obviously, the curve can be approximated by Normal distribution. In Eq. (36), we are concerned with lower limit for the population mean $\mu$ since 
the upper limit of SIR is confided. To construct a one-sided confidence interval, we consider the area in only one tail of a standard Normal distribution. We suppose that the mean unknown. Therefore,

$$
\begin{aligned}
P(z \geq Z) & =P\left(z \geq \frac{M_{n}-\mu}{\sigma / \sqrt{n}}\right) \\
& =P\left(M_{n}-z \cdot \frac{\sigma}{\sqrt{n}} \leq \mu\right) \\
& =P\left(L_{1} \leq \mu\right)
\end{aligned}
$$

where $Z=\frac{M_{n}-\mu}{\sigma / \sqrt{n}}$ is the zero mean, unit-variance random variable. The lower limit $L_{1}=$ $M_{n}-z \cdot \frac{\sigma}{\sqrt{n}}$. The $(1-\alpha) \times 100 \%$ confidence interval for the mean $\mu$ is $\left[M_{n}-z \cdot \frac{\sigma}{\sqrt{n}}, \infty\right]$.

When computing confidence intervals for an unknown population mean $\mu$, we have always assumed that $\sigma$, the population standard deviation, is known. In reality, this is unlikely to be the case; if $\mu$ is unknown, then $\sigma$ is probably unknown as well. Instead of involving the normal distribution, however, the analysis depends on a new probability distribution known as Student's t distribution [24].

Since $\sigma$ is unknown, so $Z=\frac{M_{n}-\mu}{\sigma / \sqrt{n}}$ can't be used anymore. Instead, we use

$$
t=\frac{M_{n}-\mu}{V_{n} / \sqrt{n}}
$$

where $V_{n}$ is the sample standard deviation in Eq. (35). If $X_{j}$ is normal distribution, $j=1$, $\ldots, n$, then $t$ has a $t$ distribution with $n-1$ (df) degrees of freedom, denoted by $t_{n-1}$.

The $t$ distribution is very similar to the normal distribution. For large degrees of freedom, the $t$ distribution and the normal distribution are equal. Figure 13 compares the $t$ distribution with different "degrees of freedom" to the normal distribution. With 5 degree of freedom, the $t$ distribution is fairly different from the normal distribution, but for more than about 15 degrees of freedom, they are very similar. Then the $(1-\alpha) \times 100 \%$ confidence interval for $\mu$ is defined by the lower $\left(L_{1}\right)$ bound:

$$
L_{1}=M_{n}-\left(t_{n-1}\right) \frac{V_{n}}{\sqrt{n}}
$$

where $t_{n-1}$ is $t$ distribution with $n-1$ degrees of freedom. If you don't have access to a statistics program, you can find tabulated values of $t$ in most statistics texts [24]. The $(1-\alpha) \times 100 \%$ confidence interval is modified as

$$
\left[M_{n}-\left(t_{n-1}\right) \frac{V_{n}}{\sqrt{n}}, \infty\right]
$$

Finally, we use the simulation result to prove the DTI. However, we notice that a false alarm will caused in the decision mechanism since the fluctuation of SIR is large from the simulation results of Fig. 14. Therefore, we must modify our DTI to avoid false alarm. We can modify the decision mechanism as follows:

Step 1. The concept of moving average is adopted by SIR to prevent fluctuation of performance. We have

$$
S I R^{\prime}=\frac{1}{n} \sum_{j=0}^{n-1} S I R(i-j)
$$


Step 2. To prevent the lower bound which is varied with the changed environment. The lower bound is modified as

$$
L_{1}^{\prime}(i)=\frac{L_{1}+L_{1}^{\prime}(i-1)}{2}
$$

where the $L_{1}^{\prime}$ initial value is $L_{1}$.

With the combination of DTI and subspace-based blind multiuser detection, as shown in Fig. 1, the changed environment can be efficiently detected. For example, at first the SIR can be acquired by the subspace-based blind multiuser detection and substitution the SIR into Eq. (40-42) to determine that if the environment is changed. The environment is assumed to be changed if the SIR is not exist within interval, therefore, the signal subspace components are reset and replaced by the new signal subspace which is reacquired by applying a SVD method.

\section{Simulation and Discussion}

In the following simulations, we adopt the MRC-Rake receiver with subspace- based blind adaptive linear MMSE detector over a time-varying channel. The receiver that combinations the DTI and subspace-based blind multiuser detection is shown in Fig. 1. Some parameters used in this experiment are as follows: $S N R_{k}=10 \log \left(E_{k} / \sigma^{2}\right)$; the number of pulses representing one information symbol is $N_{f}=8$; the number of chips per frame is $N_{c}=16$; the number of user is $K=6$; the desired user is user 1 and all users have the same transmitted energy. The signal-to-noise ratio is $20 \mathrm{~dB}$. Assume that the signal is first (i.e. $t=1)$ transmitted through the CM1, and then change to CM4 at $\mathrm{t}=2,001$. Table 2 lists the experimental results of 1,000 times which are obtained from the method of DTI. In Table 2, the table contains many states such as miss (not detect), false alarm $(t=1-2,000)$, and correct detection $(t=2,001-$ $4,000)$. Besides, the correct detection also divides into rapid detection $(t=2,001-2,050)$ and slow detection $(t>2,051)$. The data in Table 2 is affected by the parameter, i.e. the sample size $\mathrm{n}$. The false alarm will decrease when the sample size increase. Apparently, the probability of decision error can be reduced with $n=30$. But when $n>30$, the probability of slow detection is increase. The results exhibit optimal trade-off between detect time and sample size in Table 2. As noted based on Table 2, the performance trend is sustained, where the $n=30$ outperforms the other consider sample size, regard of the time-varying channel. We will discuss the results which may be caused by the DTI decision error $(t=1-2,000)$ or slowly decision $(t>2,050)$.

Table 2 The experimental results are obtained from the method of decision timing instant over time-varying channel (CM1/CM4)

\begin{tabular}{lllll}
\hline $\begin{array}{l}n \text { (ample } \\
\text { size) }\end{array}$ & $\begin{array}{l}\text { Detect Time (miss) } \\
\text { (not detect) }(\%)\end{array}$ & $\begin{array}{l}\text { Detect Time (false } \\
\text { alarm) } \mathrm{t}=1-2,000\end{array}$ & $\begin{array}{l}\text { Detect Time (rapid) } \\
\mathrm{t}=2,001-2,050\end{array}$ & $\begin{array}{l}\text { Detect Time (slow) } \\
\mathrm{t}>2,050\end{array}$ \\
\hline 10 & 0 & $0.2 \%(1,975)$ & $99.6 \%(2,009.1)$ & $0.2 \%(2,132)$ \\
20 & 0 & $0.2 \%(1,863)$ & $99.7 \%(2,013.3)$ & $0.1 \%(2,053)$ \\
$\mathbf{3 0}$ & $\mathbf{0}$ & $\mathbf{0} \%$ & $\mathbf{9 9 . 9} \%(2,017.6)$ & $\mathbf{0 . 1} \%(2,067)$ \\
40 & 0 & 0 & $99.8 \%(2,021.4)$ & $0.2 \%(2,153)$ \\
50 & 0 & 0 & $99.8 \%(2,025.3)$ & $0.2 \%(2,146.5)$ \\
\hline
\end{tabular}

ps. (•) is the average of detection timing 


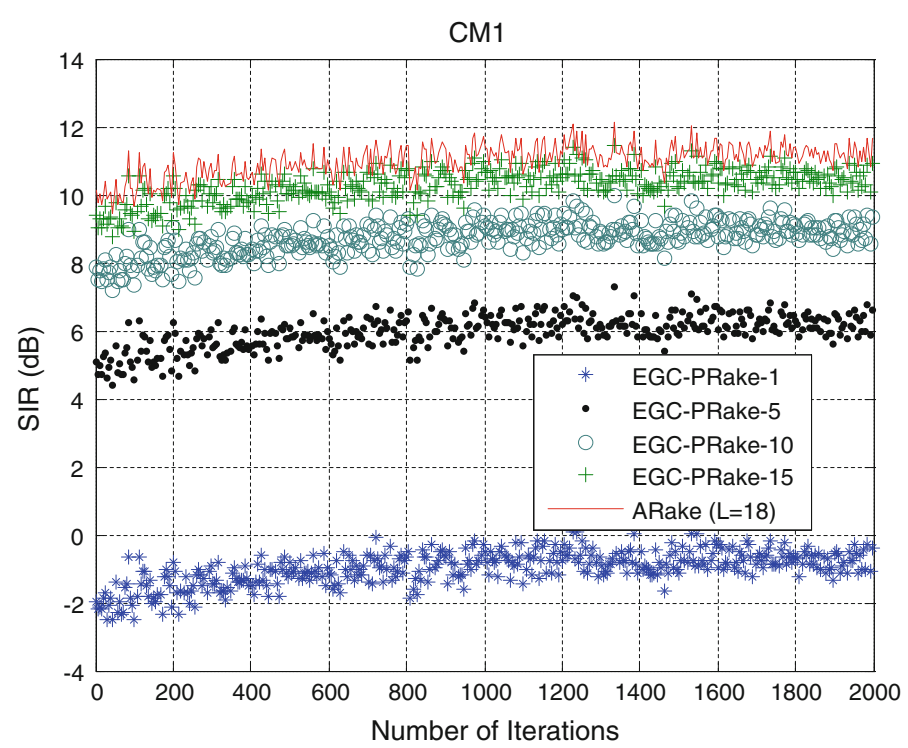

Fig. 2 Performance of the subspace-based blind MMSE detector with EGC-Prake (CM1)

Figure 2 shows the performance of the EGC-Prake under the CM1 with different number of fingers. We observe that the performance has a significant improve with a number of 10 fingers. With a number of 15 fingers, the performance is close to the EGC-Arake that receives the total transmitted energy; however, the computation complexity is increased as well. Hence, there may be a trade-off between the system performance and computation complexity. The performance analysis of the subspace-based blind MMSE detector with EGC-Prake under the CM1 that considers the ISI and non-ISI situations is demonstrated in Fig. 3 Simulation result shows that the influence of ISI can be neglected in LOS (line-of-sight) since the stronger component of channel gains are collected in the first few paths and therefore the subsequent paths only have a slight affect in system performance. The performance comparison of MRC-Arake and EGC-Arake receiver is shown in Fig. 4. We notice that if the channel coefficient is perfectly estimated, the MRC-Arake receiver can perform a better performance than EGC-Arake receiver. Nevertheless, for the practical system, it is difficult to estimate the channel coefficient perfectly and will result in a poor performance of the MRCArake receiver. Therefore, the EGC-Rake receiver which does not need to estimate channel coefficients is perfected. Figures 5 and 6 show the performance of SIR in NLOS (non-lineof-sight). Compared with CM1, the simulation results indicate that the system performance is severely affected by the multipath interference.

Figures 7, 8, 9, 10 and 11 shows the MRC-Rake receiver without considering the DTI over a time-varying channel. The simulation result illustrates that the performance which is suffered from a changed environment at $\mathrm{t}=2$, 001. After $\mathrm{t}=2,001$, the SIR need some time to converge to steady-state. This is because that the subspace tacking algorithm can not update the signal subspace components rapidly under the suddenly changed environment. Figures 7, 8, 9, 10 and 11 show different SIR under different environment changes, i.e., CM1-CM4, CM4-CM1, CM1-CM1 (different channel coefficient), CM1-CM2 and CM1-CM3.

Figure 15 shows relationship between SIR of MRC-Rake receiver with DTI and lower bound for $99 \%$ confidence interval. The gray curve is the SIR of MRC-Rake receiver with 


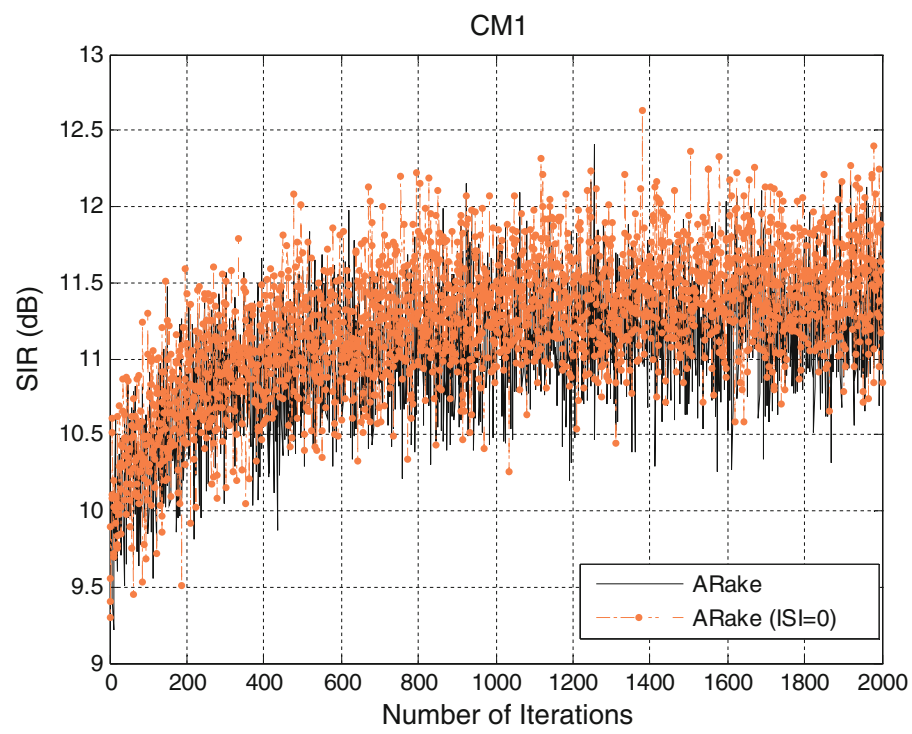

Fig. 3 Performance of the subspace-based blind MMSE detector with EGC-Arake (CM1)

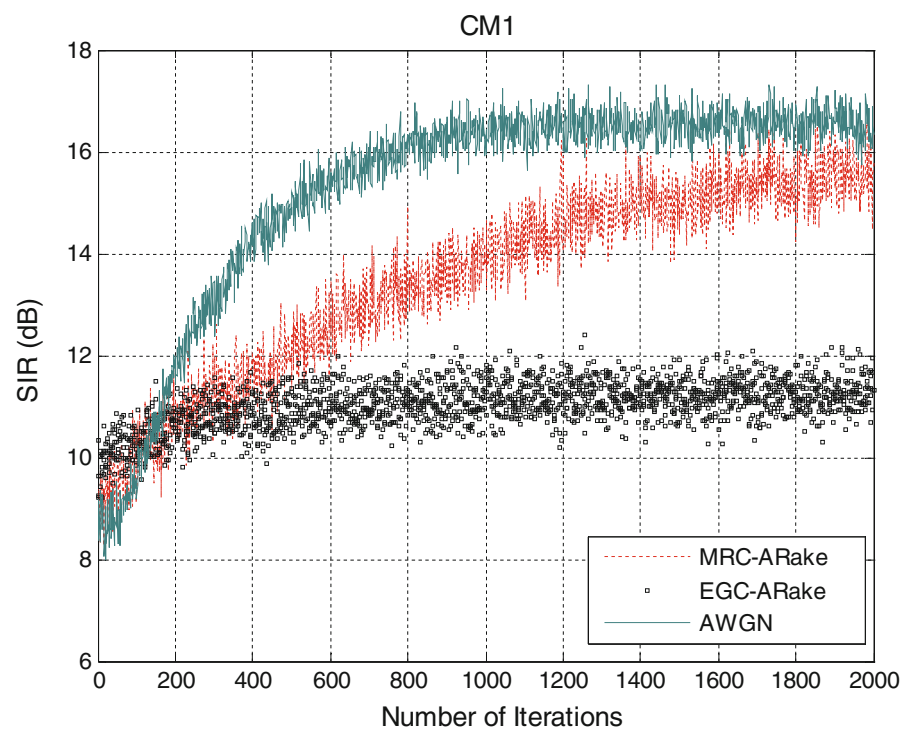

Fig. 4 Performance comparison of the subspace-based blind MMSE detector with EGC-Arake and MRCArake (CM1)

the DTI; the blue curve is the SIR' from the modified SIR; the pink curve is the lower bound $L_{1}$ of confidence interval; the yellow curve is $L_{1}^{\prime}$ from the modified lower bound $L_{1}$. The data plotted is the one-run simulation result. Compare the $S I R$ with $L_{1}$, we notice that serious false alarm will caused since the fluctuation of SIR is large. Therefore, we modify the SIR and $L_{1}$ to perform the DTI. The $S I R$ ' and $L_{1}^{\prime}$ are modified form the SIR and $L_{1}$, respectively. Compare the SIR' with $L_{1}^{\prime}$, the environment is assumed to be changed if the SIR' is lower 




Fig. 5 Performance of the subspace-based blind MMSE detector with EGC-Prake (CM4)

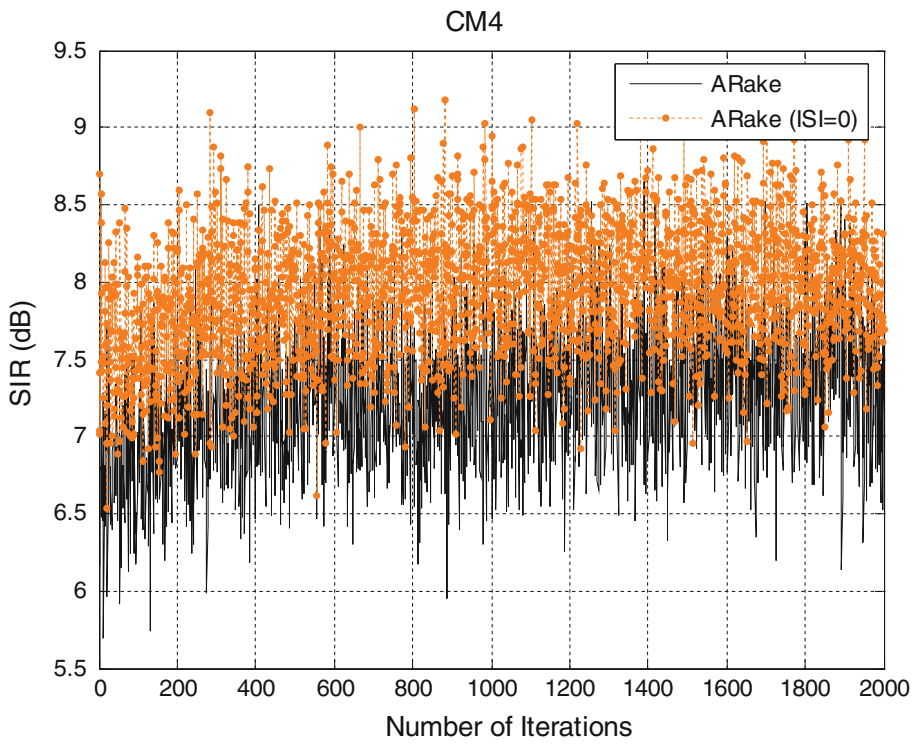

Fig. 6 Performance of the subspace-based blind MMSE detector with EGC-Arake (CM4)

than $L_{1}^{\prime}$. Therefore, simulation result shows that the DTI can correctly detect the changing environment at $t=2,013$ (Figs. 12, 13, 14).

The simulation results of one run are shown in Figs. 15 and 16. Figure 15 indicates the MRC-Rake receiver without considering the DTI over a time-varying channel. Figure 16 shows the MRC-Rake receiver with the DTI over time-varying channel. Obviously, the performances in Figs. 15 and 16 both have serious fluctuation. Besides, compare Fig. 15 with 




Fig. 7 Performance of the MRC-Rake receiver without considering the decision timing instant over timevarying channel. The simulation starts $(\mathrm{t}=0)$ in the $\mathrm{CM} 1$ channel; at $\mathrm{t}=2,001$ in the $\mathrm{CM} 4$ channel. The data plotted is the average over 1,000 runs

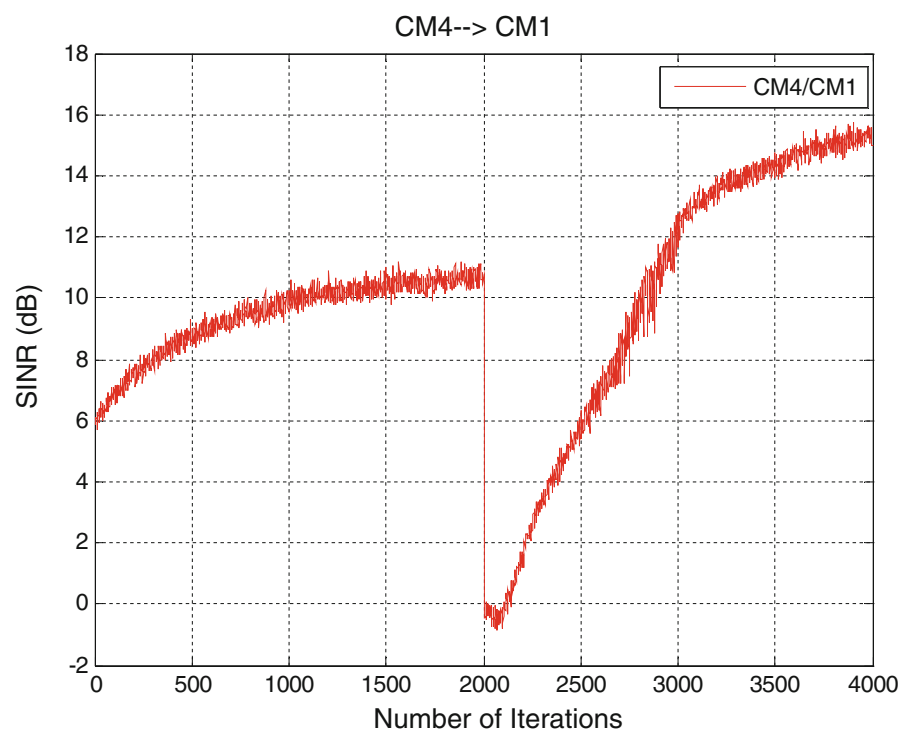

Fig. 8 Performance of the MRC-Rake receiver without considering the decision timing instant over timevarying channel. The simulation starts $(\mathrm{t}=0)$ in the $\mathrm{CM} 4$ channel; at $\mathrm{t}=2,001$ in the $\mathrm{CM} 1$ channel. The data plotted is the average over 1,000 runs

Fig. 16, the variation environment can be detected by DTI at $t=2,013$ in Fig. 16. Therefore, it is obvious that the DTI has a faster convergence speed even through the fluctuation of SIR is serious. 


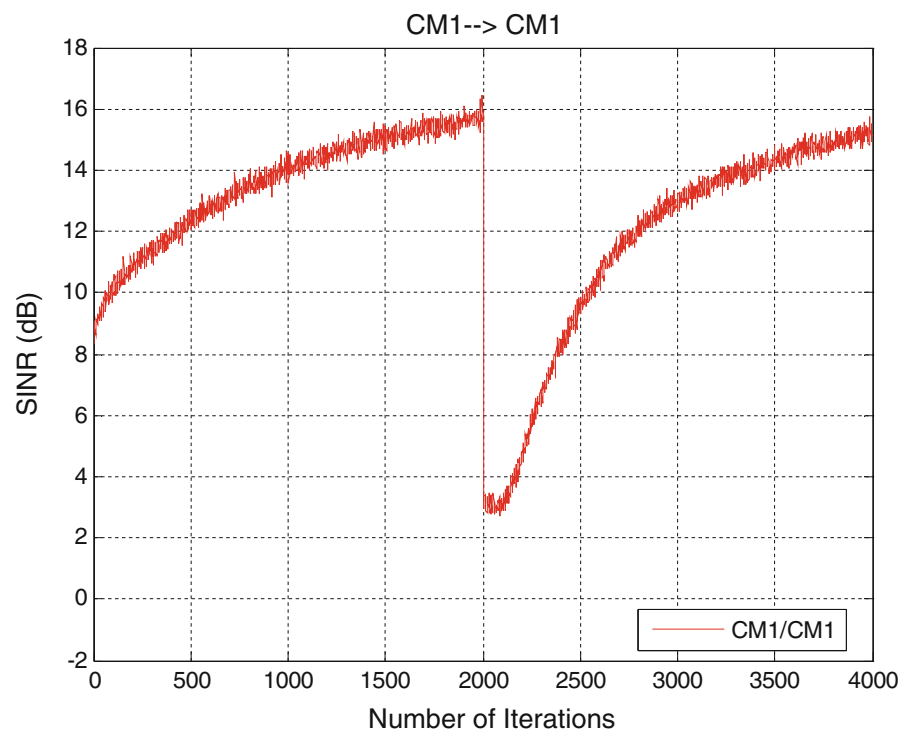

Fig. 9 Performance of the MRC-Rake receiver without considering the decision timing instant over timevarying channel. The simulation starts $(\mathrm{t}=0)$ in the $\mathrm{CM} 1$ channel; at $\mathrm{t}=2,001$ in the CM1 channel with different channel coefficients. The data plotted is the average over 1,000 runs

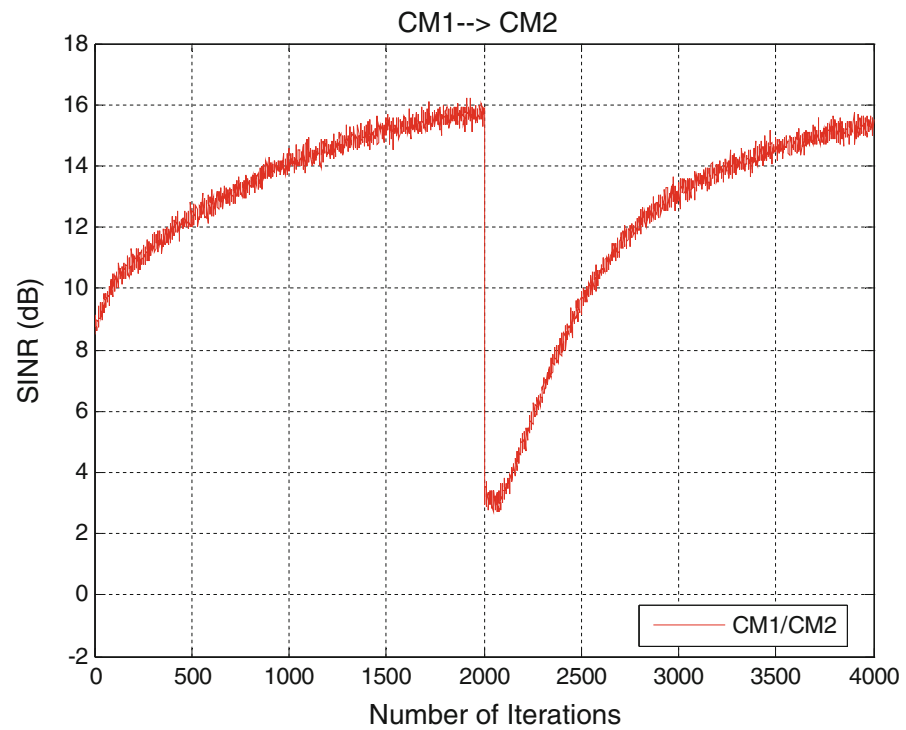

Fig. 10 Performance of the MRC-Rake receiver without considering the decision timing instant over timevarying channel. The simulation starts $(\mathrm{t}=0)$ in the $\mathrm{CM} 1$ channel; at $\mathrm{t}=2,001$ in the $\mathrm{CM} 2$ channel. The data plotted is the average over 1,000 runs

From Figs. 15 and 18 indicates the simulation result when a false alarm is occurred, the false alarm is detected by the DTI at $t=514$. Therefore, the system will reset the eigencomponents and provide new eignecompoments by recollect new 50 data. Notice that the DTI is stops running while the data are recollecting. At $t=2,059$, the changing environment is still 


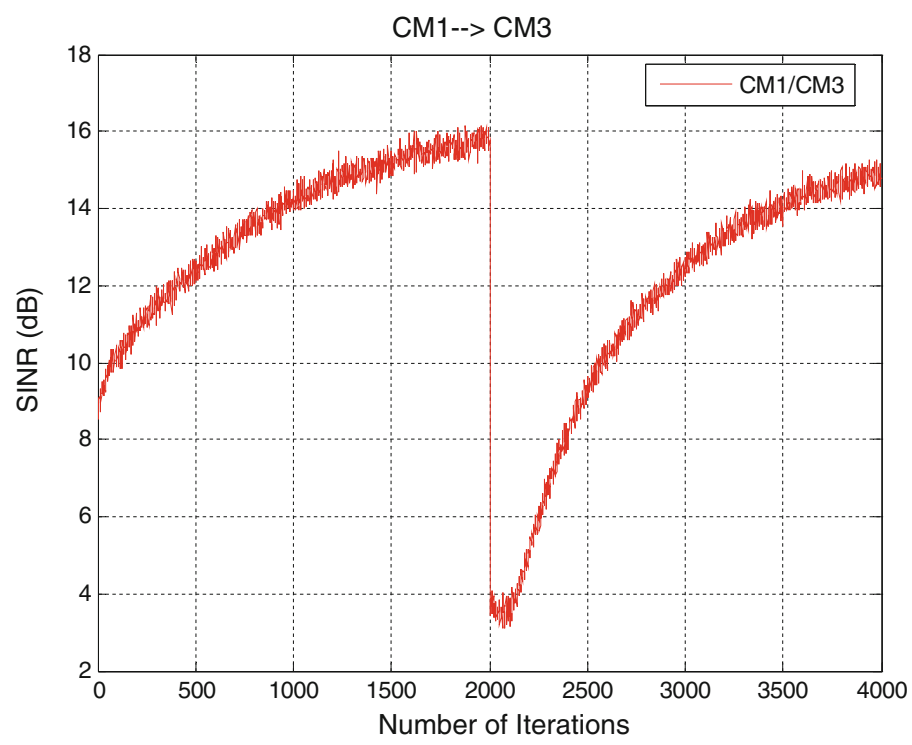

Fig. 11 Performance of the MRC-Rake receiver without considering the decision timing instant over timevarying channel. The simulation starts $(\mathrm{t}=0)$ in the $\mathrm{CM} 1$ channel; at $\mathrm{t}=2,001$ in the $\mathrm{CM} 3$ channel. The data plotted is the average over 1,000 runs

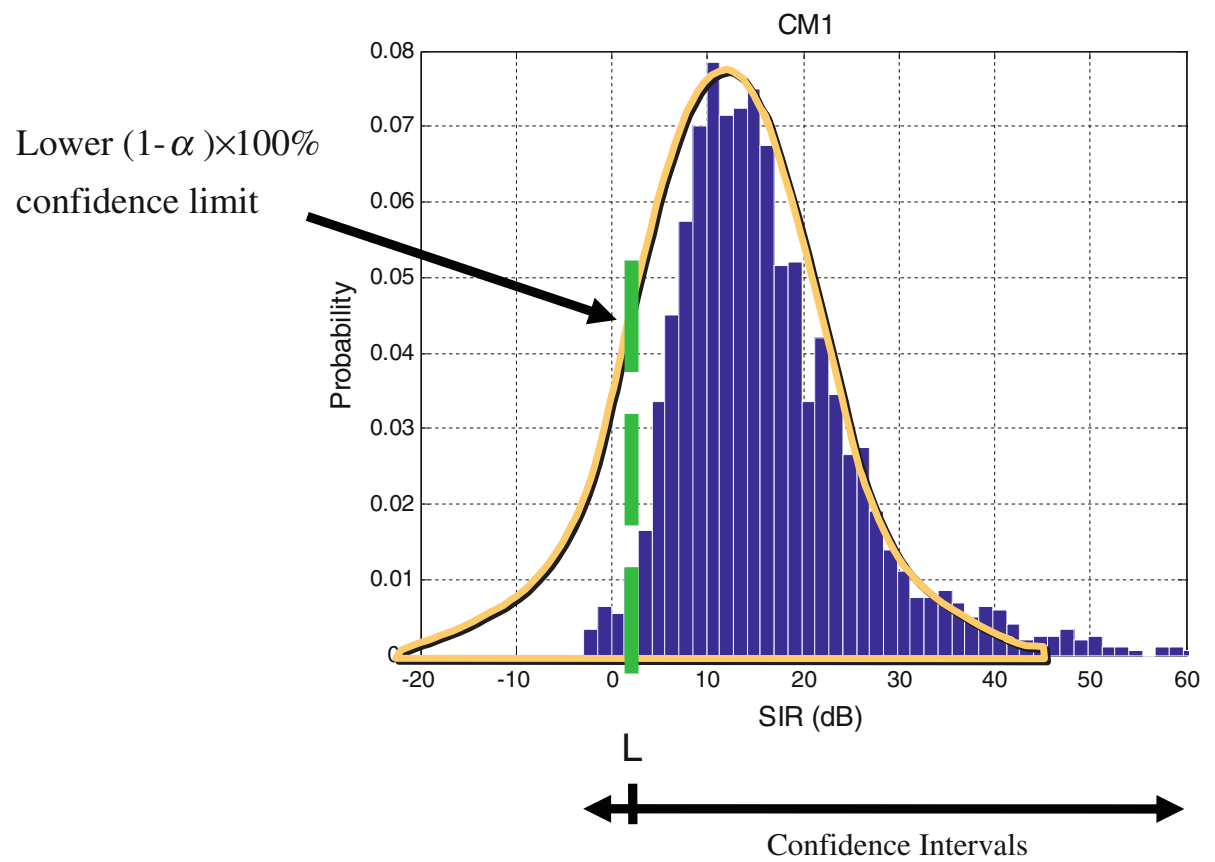

Fig. 12 Histogram from the SIR

detected by the DTI. Hence, we conclude that the false detect has no effect on system performance due to the new eigencomponents are acquired from the original environment. Figures 19,21 and 22 demonstrate the benefit of the DTI which can offer a rapidly convergence 


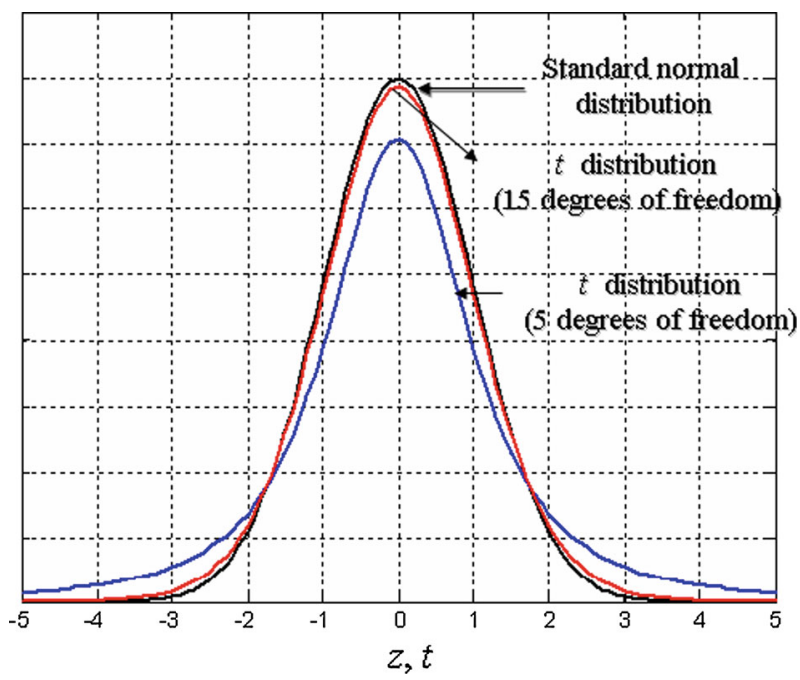

Fig. 13 Standard normal distribution and Student's $t$ distribution

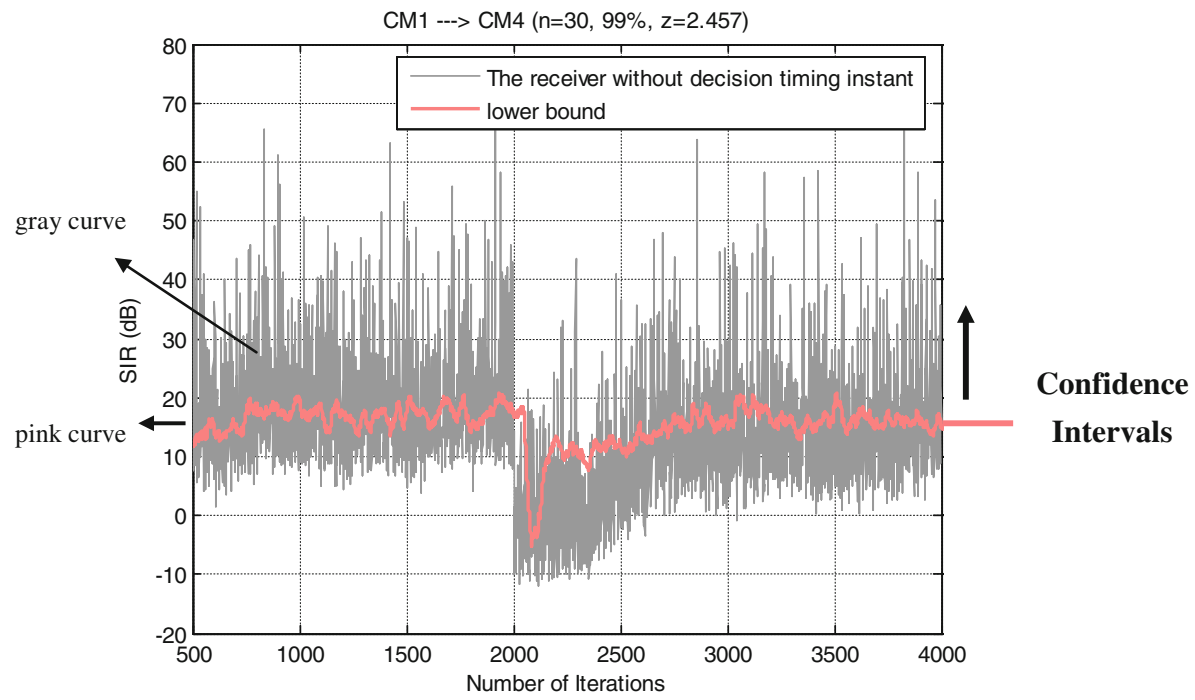

Fig. 14 Plot the $99 \%$ confidence interval (pink curve). The gray curve is the simulation result of MRC-Rake receiver without considering the decision timing instant over time-varying channel. The simulation starts $(\mathrm{t}=0)$ in the CM1 channel; at $\mathrm{t}=2,001$ in the CM4 channel. The data plotted is the one-run simulation result. (Color figure online)

speed and decrease the loss of symbols. To pay attention to Fig. 22, as compare with Figs. 19, 20 and 21 , the system which is suffered by a serious variation in transmission environment can perform a significant improvement (Fig. 17).

\section{Concluding Summary and Discussion}

In this paper, we proposed blind multiuser methods based on DTI and MMSE procedures. Our novel multiuser detector matched to the DTI has great potential for deployment in future 


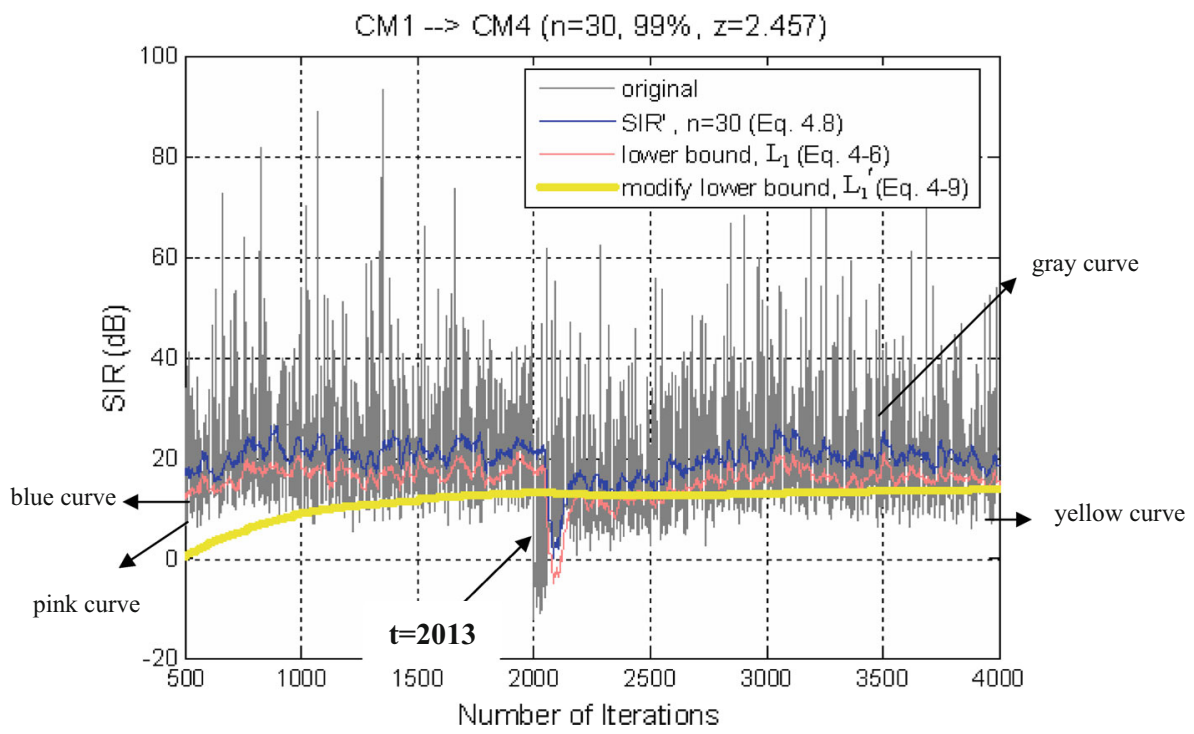

Fig. 15 Relationship between SIR of MRC-Rake receiver with DTI and lower bound for $99 \%$ confidence interval. The simulation starts $(\mathrm{t}=0)$ in the CM1 channel; at $\mathrm{t}=2,001$ in the CM4 channel $(n=30)$. The data plotted is the one-run simulation result (timing $=2,013$ )



Fig. 16 Performance of the MRC-Rake receiver without the decision timing instant over time-varying channel. The simulation starts $(\mathrm{t}=0)$ in the CM1 channel; at $\mathrm{t}=2,001$ in the CM4 channel. The data plotted is the one-run simulation result

generation systems. Furthermore, a major advantage of the DTI algorithm is that it admits an adaptive implementation with low computational complexity instead of SVD. In addition, simulations and comparisons confirm that the proposed approach is superior to [23], the main difference is that we adopt a Rake receiver scheme to combat against the multipath fading 


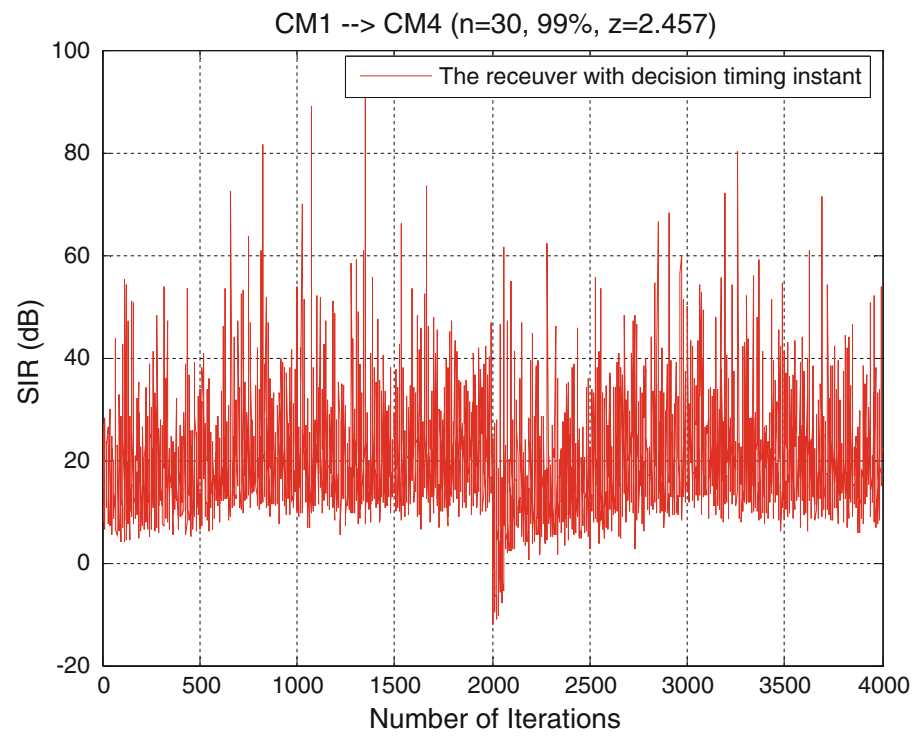

Fig. 17 Performance of the MRC-Rake receiver with the decision timing instant over time-varying channel. The simulation starts $(\mathrm{t}=0)$ in the CM1 channel; at $\mathrm{t}=2,001$ in the CM4 channel. The data plotted is the one-run simulation result (timing $=2,013$ )

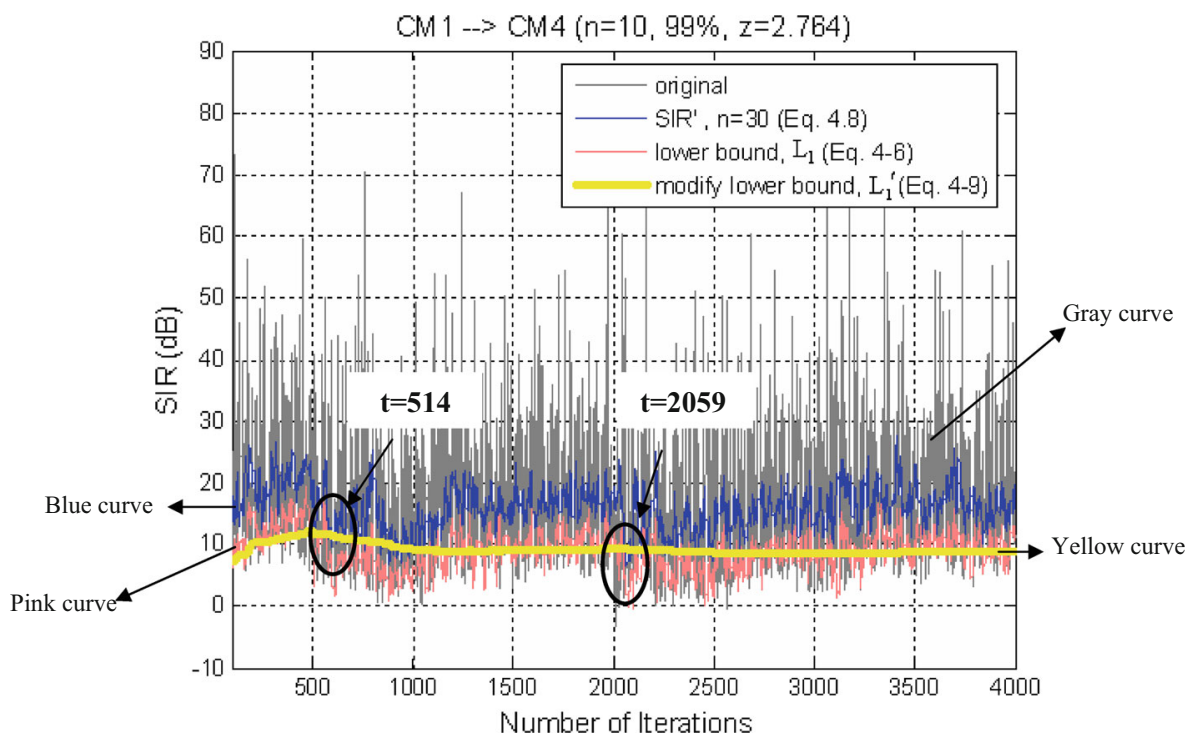

Fig. 18 Illustration of the false alarm being occurred (timing $=514)$. The simulation starts $(t=0)$ in the CM1 channel; at $\mathrm{t}=2,001$ in the CM4 channel $(n=10)$. The data plotted is the one-run simulation result

channel. In general, Rake receiver performs better than MMSE methods. But they require higher complexity and their performance degrades more rapidly with increase of total number of users. If complexity is a major concern in real applications, DTI are good choices because of their relatively low complexity and highly tolerable to number of simultaneous users. 


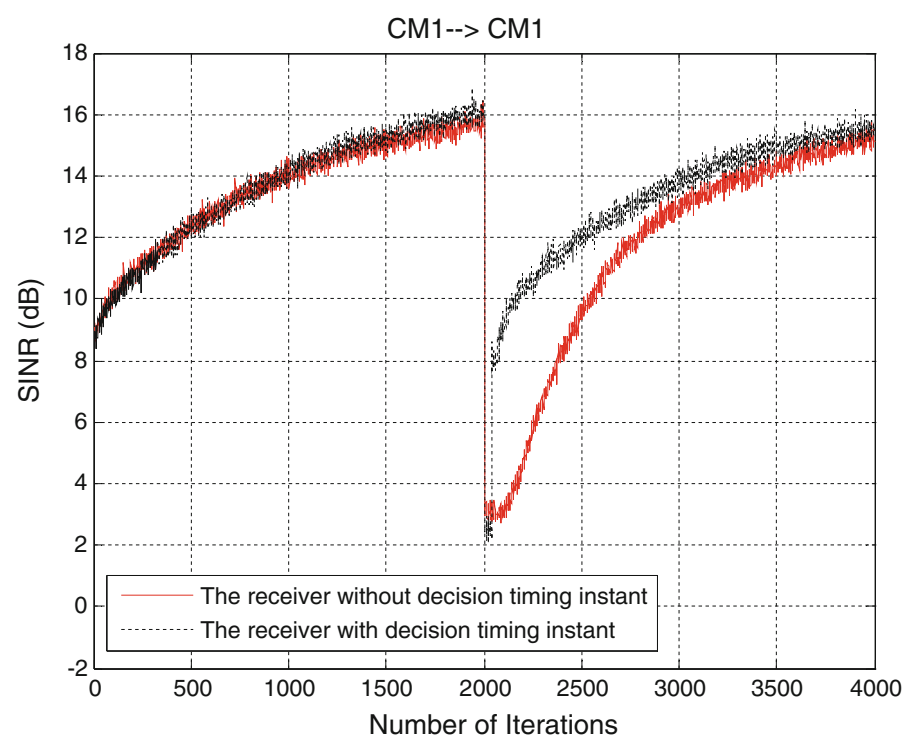

Fig. 19 Performance of the MRC-Rake receiver over time-varying channel. The simulation starts $(t=0)$ in the CM1 channel; at $\mathrm{t}=2,001$ in the CM1 channel with different channel coefficients $(n=30)$. The data plotted is the average over 1,000 runs

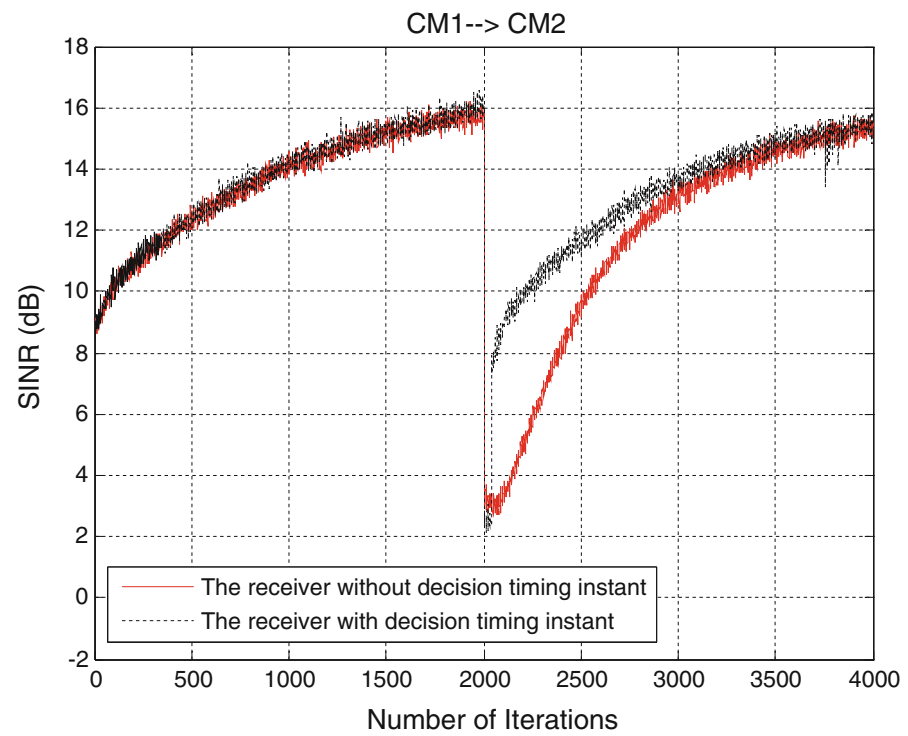

Fig. 20 Performance of the MRC-Rake receiver over time-varying channel. The simulation starts $(t=0)$ in the CM1 channel; at $\mathrm{t}=2,001$ in the CM2 channel $(n=30)$. The data plotted is the average over 1,000 runs

Simulation results that the performance is improved by increasing the number of fingers of a Rake receiver, because more transmitted energy can be captured. Besides, compared the simulation results of CM1 with CM4, we find that the system performance in CM4 is much worse than that in CM1 due to the serious multipath interference. Another important 




Fig. 21 Performance of the MRC-Rake receiver over time-varying channel. The simulation starts $(t=0)$ in the CM1 channel; at $\mathrm{t}=2,001$ in the CM3 channel $(n=30)$. The data plotted is the average over 1,000 runs

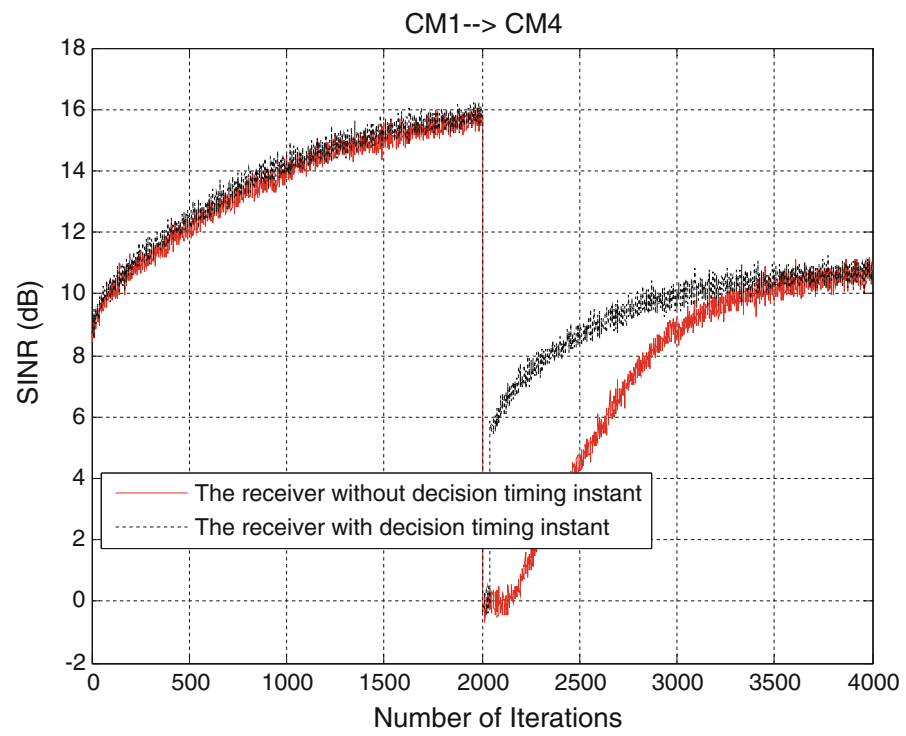

Fig. 22 Performance of the MRC-Rake receiver over time-varying channel. The simulation starts $(\mathrm{t}=0)$ in the CM1 channel; at $\mathrm{t}=2,001$ in the CM4 channel $(n=30)$. The data plotted is the average over 1,000 runs

implementation advantage is that results demonstrate the fast tracking capability and high steady-state estimation accuracy of the proposed algorithm in both the time-invariant and time-variant environments.

Moreover, this paper presents with changing the transmission positions rapidly, it is equivalent to suffer the transition of the channel models, due to the different transmission distances 
or characteristics. It results in large performance degradation and a slow convergence speed to the steady state. We demonstrated that the proposed DTI method is able to detect the variation of channel environment fast and precisely, therefore, it can improve both the system performance and the efficiency of convergence speed. In fact, the DTI method can be applied not only to UWB system but also to any other systems (for example: mobile systems, DAB, DVB) which require to detect the sudden change of the environment. These interesting topics will be further investigated in the future.

Open Access This article is distributed under the terms of the Creative Commons Attribution License which permits any use, distribution, and reproduction in any medium, provided the original author(s) and the source are credited.

\section{References}

1. Win, M. Z., \& Scholtz, R. A. (1998). Impulse radio: How it works. IEEE Communications Letters, 2(2), 36-38.

2. Yang, L., \& Giannakis, G. B. (2004). Ultra-wideband communications: An idea whose time has come. IEEE Signal Processing Magazine, 21(6), 26-54.

3. Bellusci, Giovanni, Janssen, Gerard J. M., Yan, Junlin, \& Tiberius, Christian C. J. M. (2012). Performance evaluation of a low-complexity receiver concept for TOA-based ultrawideband ranging. IEEE Transactions on Vehicular Technology, 61, 3825-3837.

4. Federal Communications Commission. (2002, April). Revision of part 15 of the commission's rules regarding ultra-wideband transmission systems, First Report and Order, ET Docket, pp. 98-153.

5. Reggiani, L., \& Maggio, G. M. (2005). Rapid search algorithms for code acquisition in UWB impulse radio communications. IEEE Journal on Selected Areas in Communications, 23(5), 898-908.

6. Salih-Alj, Y., Despins, C., \& Affes, S. (2011). Design considerations for an UWB computationallyefficient fast acquisition system for indoor line-of-sight ranging applications. IEEE Transactions on Wireless Communications, 10(8), 2776-2784.

7. Navarro, M., \& Najar, M. (2011). Frequency domain Joint TOA and DOA estimation in IR-UWB. IEEE Transactions on Wireless Communications, 10(10), 1-11.

8. Suwansantisuk, W., \& Win, M. Z. (2007). Multipath aided rapid acquisition: Optimal search strategies. IEEE Transactions on Information Theory, 53(1), 174-193.

9. He, N., \& Tepedelenlioglu, C. (2007). Fast and low-complexity frame-level synchronization for transmitted reference receivers. IEEE Transactions on Wireless Communications, 6(3), 1014-1023.

10. Di Renzo, M., et al. (2008). A novel class of algorithms for timing acquisition of differential transmitted reference UWB receivers: Architecture, performance analysis and system design. IEEE Transactions on Wireless Communications, 7(6), 2368-2387.

11. Nerguizian, C. (2003, September) Radiolocation in an underground mining environment, Ph.D. Dissertation. INRS-EMT, Montreal, Canada.

12. Homier, E. A., \& Scholtz, R. A. (2002). Rapid acquisition of ultra-wideband signals in the dense multipath channel. in Proceedings of IEEE Conference. Baltimore, MD: UWB Systems \& Technologies.

13. Lee, J. Y., \& Scholtz, R. A. (2002). Ranging in a dense multipath environment using a UWB radio link. IEEE Journal on Selected Areas in Communications, 20(12), 1677-1683.

14. Blazquez, R., Newaskar, P., \& Chandrakasan, A. (2003). Coarse acquisition for ultra wideband digital receivers. In Proceedings of International Conference on Acoustics, Speech, and Signal Processing, Hong Kong, 137-140.

15. Maravic, I., Kusuma, J., \& Vetterli, M. (2003). Low-sampling rate UWB channel characterization and synchronization. Journal of Communications and Networks, 5(4), 319-327.

16. Carbonelli, Cecilia, \& Mengali, Umberto. (2006). Synchronization algorithms for UWB signals. IEEE Transactions on Communications, 54(2), 329-338.

17. Carbonelli, C., Franz, S., Mengali, U., \& Mitra, U. (2004). Semi-blind ML synchronization for UWB transmitted reference systems. Conference on Signals, Systems and Computers, 2, 1491-1495.

18. Lottici, V., D' andrea, A., \& Mengali, U. (2003). Channel estimation for ultrawideband communications. IEEE Journal on Selected Areas in Communications, 20(9), 1638-1645.

19. Yang, L., \& Giannakis, G. B. (2005). Timing ultra-wideband signals with dirty templates. IEEE Transactions on Communications, 53(11), 1951-1963. 
20. Homier, E. A., \& Scholtz, R. A. (2003). A generalized signal flow graph approach for hybrid acquisition of ultra-wideband signals. International Journal of Wireless Information Networks, 10(4), 179-191.

21. Guvenc, I., \& Sahinoglu, Z. (2005). Threshold selection for UWB TOA estimation based on kurtosis analysis. IEEE Communications Letters, 9(12), 1025-1027.

22. Dardari, D., Chong, C.-C., \& Win, M. Z. (2008). Threshold-based time-of arrival estimators in UWB dense multipath channels. IEEE Transactions on Communications, 56(8), 1366-1378.

23. Wand, X., \& Poor, H. V. (1998). Blind multiuser detection: A subspace approach. IEEE Transactions on Information Theory, 44, 677-690.

24. Leon-Garcia, A. (1994). Probability and random processes for electrical engineering (2nd ed.). Reading: Addison-Wesley.

25. Saleh, A., \& Valenzuela, R. (1987). A statistical model for Indoor multipath propagation. IEEE Journal on Selected Areas in Communications, SAC-5(2), 128-137.

26. Foerster, J., et al. (2003, February). Channel modeling sub-committee report final. IEEE 802. 15 Working Group for Wireless Personal Area Networks (WPANs), IEEE P802.15-02/490r1-SG3a.

27. Cassioli, D., Win, M. Z., Vatalaro, F., \& Molisch, A. F. (2002). Performance of low-complexity RAKE reception in a realistic UWB channel. Proceedings of the IEEE International Conference on Communications, 2, 763-767.

28. Brennan, D. (1959). Linear diversity combining techniques. Proceedings of the IRE, 47, 1075-1102.

29. Fishler, E., Poor, H. V. (2002). On the tradeoff between two types of processing gain. In Proceedings of the 40th Annual Allerton Conference on Communication, Control, and Computing Monticello, IL, 2-4 October, 2002.

30. Yang, B. (1995). Projection approximation subspace tracking. IEEE Transactions on Signal Processing, 44, 95-107.

31. Moulines, E., Duhamel, P., Cardoso, J. F., \& Mayrargue, S. (1995). Subspace method for the blind identification of multichannel FIR filters. IEEE Transactions on Signal Processing, 43(2), 516-525.

32. Wang, X., \& Poor, H. V. (1998). Blind equalization and multiuser detection in dispersive CDMA channels. IEEE Transactions on Communications, 46(1), 91-103.

33. Yang, B. (1995). An extension of the PASTd algorithm to both rank and subspace tracking. IEEE Signal Processing Letters, 2, 179-182.

34. Honig, M., Madhow, U., \& Verdú, S. (1995). Blind adaptive multiuser detection. IEEE Transactions on Information Theory, 41(4), 944-960.

\section{Author Biographies}

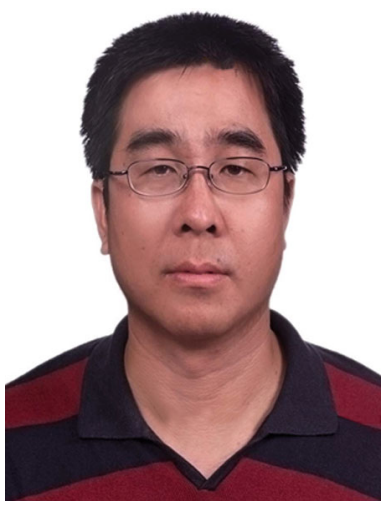

Ho-Lung Hung received the M.S. degree in electrical engineering from University of Detroit Mercy, Michigan, USA, in 1994 and the Ph.D. degree in electrical engineering from National Chung Cheng University, Chia-Yi, Taiwan, in 2007. From 1995 to 2006, he was a lecturer with the Department of Electrical Engineering, Chienkuo Technology University, Taiwan. Since 2007, he was an associate professor with the Department of Electrical Engineering, Chienkuo Technology University, Taiwan. His current research interests are in wireless communications, detection of spread-spectrum signal, wireless sensor networks, evolutionary computation, and intelligent systems. Dr. Hung serves as an Associate Editor for the TELECOMMUNICATION SYSTEMS. 


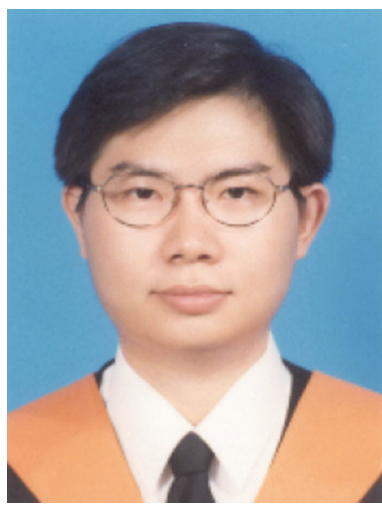

Chia-Hsin Cheng received the M.S. degree in 1997 and the Ph.D. degree in 2007, both in Electrical Engineering from the National Chung Cheng University, Chia-Yi, Taiwan. Currently, he is an Associate Professor with the Department of Electrical Engineering, National Formosa University, Yunlin, Taiwan. His research interests are in the areas of wireless sensor networks (WSNs), communication networks, interference cancellation, spread-spectrum systems, OFDM systems, and UWB system. 